\title{
ON MAPPING SEQUENCES
}

\author{
YASUTOSHI NOMURA
}

\section{Introduction}

In 1958 D. Puppe developed the theory of Abbildungsfolge and applied it to the study of Hilton formula and his sphärenähnliche Mannigfaltigkeiten [12]." We shall be concerned here with a dual situation.

In Part I we introduce the mapping sequence and discuss some applications of it. Let $f: X \rightarrow Y$ be a map ${ }^{2)}$ of one topological space to another. We associate with it the following two sequences which are homotopically equivalent to each other $(\$ 2, T h .2)$

$$
\begin{aligned}
& \mathfrak{M}_{f}: \cdots \rightarrow \Omega^{2} X \stackrel{\Omega^{2} f}{\longrightarrow} \Omega^{2} Y \stackrel{\Omega I f}{\longrightarrow} \Omega E_{f} \stackrel{\Omega P f}{\rightarrow} \Omega X \stackrel{\Omega f}{\rightarrow} \Omega Y \stackrel{I f}{\rightarrow} E_{f} \stackrel{P f}{\longrightarrow} X \stackrel{f}{\rightarrow} Y, \\
& \mathfrak{P} f: \cdots \rightarrow E_{P^{5} f} \stackrel{P^{6} f}{\longrightarrow} E_{P^{4} f} \stackrel{P^{5} f}{\longrightarrow} E_{P^{3} f} \stackrel{P^{4} f}{\rightarrow} E_{P^{2} f} \stackrel{P^{3} f}{\longrightarrow} E_{P f} \stackrel{P^{2} f}{\longrightarrow} E_{f} \stackrel{P f}{\rightarrow} X \stackrel{f}{\rightarrow} Y,
\end{aligned}
$$

where $P f: E_{f} \rightarrow X$ is the fibering induced by $f$ from the contractible fibre space over $Y$, If the injection of the fibre to the total, and $\Omega$ the loop functor. Furthermore, it will be shown in $\$ 4$ that they are homotopically invariant in the sense that homotopically equivalent sequences are obtained when $f$ is altered within its homotopy class. $\mathfrak{M} f$ induces for any space $V$ an exact sequence

$(\mathfrak{M} f)_{*}: \cdots \rightarrow \pi(V, \Omega X) \stackrel{(\Omega f)_{*}}{\rightarrow} \pi(V, \Omega Y) \stackrel{(I f)_{*}}{\rightarrow} \pi\left(V, E_{f}\right) \stackrel{(P f)_{*}}{\rightarrow} \pi(V, X) \stackrel{f_{*}}{\rightarrow} \pi(V, Y)$, where $\pi(V, X)$ denotes the set of homotopy classes of maps $V \rightarrow X$. This is reduced to the usual exact sequence of nomotopy groups in case $V$ is a sphere and $f$ an inclusion.

It will turn out in $\S 5$ that $\mathfrak{M} f$, together with its invariance, gives some delicate informations about homotopy equivalences. For instance, we prove that the fibre of a contractible fibering has the same homotopy type as the

Received May 18, 1960.

1) Numbers in square brackets refer to the papers listed at the end.

2) By the term "map" is always meant a continuous mapping. 
loop space of its base. This is a variant of a theorem of Samelson [13].

In Part II we deal with an interrelation between our mapping sequence and Puppe's Abbildungsfolge. For any triple $X \stackrel{f}{\rightarrow} Y \stackrel{g}{\rightarrow} Z$ such that $g \circ f \simeq 0$, we construct the connecting diagram which is the basic machinary of our later investigation. Using this we define, for any map $f: X \rightarrow Y$, suspensions

$$
\sigma^{*}: \pi\left(C_{f}, V\right) \rightarrow \pi\left(S E_{f}, V\right)
$$

and

$$
\sigma_{*}: \pi\left(V, E_{f}\right) \rightarrow \pi\left(V, \Omega C_{f}\right)
$$

which may be regarded as an extension of usual cohomology and homotopy suspensions. Here $C_{f}$ is a mapping cone of $f$, and $S$ denotes the suspension functor.

We will prove isomorphism theorems concerning $\sigma^{*}$ and $\sigma_{*}(\S 9)$, as an application of which we present a detailed exposition of the Postnikov system in line with a treatment in [6]. Finally, it will be shown that the connecting diagram allows us to give a direct description of functional cohomology operations.

\section{Part I. Mapping Sequence and homotopy equivalence}

\section{Preliminaries}

1.1. We begin with some notations and conventions to be used here.

A fixed base point will always be chosen in each space and denoted by a subscript 0 : thus $x_{0} \in X, a_{0} \in A$. All maps and homotopies are to carry base points to such. The identity map of $X$ on itself is denoted by $1_{X}$ or simply by 1. Given two maps $f_{1}, f_{2}: X \rightarrow Y, f_{1} \simeq f_{2}$ means the existence of a homotopy between them. The fact that there exists a homotopy equivalence $\varphi: X \rightarrow Y$ is expressed by $\varphi: X \equiv Y$. Let $f, f^{\prime}, \varphi, \psi$ be maps such that $\varphi$ and $\psi$ are homotopy equivalences. Suppose the following diagram is commutative up to homotopy :

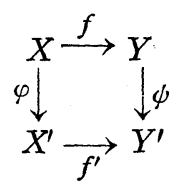

In this case we say that $f$ and $f^{\prime}$ are homotopically equivalent and we denote 
by $f \equiv f^{\prime}$.

The set of all homotopy classes of maps $X \rightarrow Y$ will be denoted by $\pi(X, Y)$, which contains the distinguished element 0 , namely, the homotopy class of the constant map $0: X \rightarrow Y$. The homotopy class of a map $f: X \rightarrow Y$ is denoted by $[f]$. Any map $g: Y \rightarrow Z$ induces a mapping $g_{*}: \pi(X, Y) \rightarrow \pi(X, Z)$ by the rule $g_{*}[f]=[g \circ f]$.

For any subspaces $A$ and $B$ of $X$ we now define $E_{A, B}(X)$ to be the path space $\{\alpha: I \rightarrow X \mid \alpha(0) \in A, \alpha(1) \in B\}$ with compact open topology, where $I$ is the unit interval $[0,1] . \quad E_{\left\{x_{0}\right\}, X}(X)$ and $E_{\left\{x_{0}\right\},\left\{x_{0}\right\}}(X)$ are abbreviated by $E X, \Omega X$ respectively. We shall denote the constant path at a point $x \in X$ by $e_{x}$. For any path $\alpha: I \rightarrow X$ let $\alpha_{a, b}: I \rightarrow X(0 \leqq a \leqq b \leqq 1)$ be a path defined by $\alpha_{a, b}(s)$ $=\alpha(a+(b-a) s)$ for $0 \leqq s \leqq 1$. Also, let $\alpha^{-1}$ be the inverse of $\alpha$, which is given by $\alpha^{-1}(s)=\alpha(1-s)$ for $s \in I$. By $\alpha \cdot \beta$ is meant the composition of $\alpha$ : $I \rightarrow X$ with $\beta: I \rightarrow X$ such that $\alpha(1)=\beta(0)$.

1.2. A map $p: X \rightarrow B$ is called a fibering if it possesses the covering homotopy property for all spaces. As indicated in W. Hurewicz [7], $D: X \rightarrow B$ is a fibering if, and only if, there exists a continuous function

$$
A:\left\{(x, \alpha) \mid x \in X, \alpha \in E_{B, B}(B), p(x)=\alpha(1)\right\} \rightarrow E_{X, X}(X),
$$

called a path lifting function for $p$, subject to the requirements $p A(x, \alpha)=\alpha$ and $\Lambda(x, \alpha)(1)=x$.

By setting

$$
\lambda(x, \alpha)=\Lambda(x, \alpha)(0),
$$

we obtain a map

$$
\lambda:\left\{(x, \alpha) \mid x \in X, \alpha \in E_{B, B}(B), p(x)=\alpha(1)\right\} \rightarrow X,
$$

with the following properties:

(i) $p \lambda(x, \alpha)=\alpha(0)$,

(ii) the map $x \rightarrow \lambda\left(x, e_{p(x)}\right), x \in X$, is homotopic to $1_{X}: X \rightarrow X$ via a homotopy which moves points along fibres.

$\lambda$ is said to be a lifting function for $p^{3{ }^{3}}$

3) It should be remarked that a map with such a $\lambda$ may fail to be a fibre map in the sense of Serre, as shown by the retraction $I \vee I \rightarrow I$. But it is sufficient for later purpose to postulate the existence of such a $\lambda$. 
Now let $f: X \rightarrow Y$ be an arbitrary map. We define $P f: E_{f} \rightarrow X$ as follows :

$$
\begin{aligned}
& E_{f}=\{(x, \beta) \mid x \in X, \beta \in E Y, f(x)=\beta(1)\}, \\
& P f(x, \beta)=x .
\end{aligned}
$$

Then $(P f)^{-1}\left(x_{0}\right)=x_{0} \times \Omega Y$. We define the injection If $: \Omega Y \rightarrow E_{f}$ by

$$
I f(\beta)=\left(x_{0}, \beta\right) \quad \text { for } \beta \in \Omega Y \text {. }
$$

Remark. In case $f$ is an inclusion then $E_{f}=E_{y_{0}, X}(Y)$. For a constant map $f(X)=y_{0}$, we have $E_{f}=X \times \Omega Y$.

Let $g: B^{\prime} \rightarrow B$ be a map and let $p: X \rightarrow B$ be a fibering. It is readily verified that if we define $p^{\prime}: X^{\prime} \rightarrow B^{\prime}$ by

$$
\begin{aligned}
& X^{\prime}=\left\{\left(b^{\prime}, x\right) \mid b^{\prime} \in B^{\prime}, x \in X, g\left(b^{\prime}\right)=p(x)\right\}, \\
& p^{\prime}\left(b^{\prime}, x\right)=b^{\prime},
\end{aligned}
$$

then $p^{\prime}: X^{\prime} \rightarrow B^{\prime}$ is also a fibering. In particular, we have

Lемма 1. Pf: $E_{f} \rightarrow X$ is a fibering whose lifting function and path lifting function are given as follows:

$$
\begin{aligned}
\lambda((x, \beta), \alpha)=\left(\alpha(0), \beta \cdot(f \alpha)^{-1}\right) & \\
& \text { for }(x, \beta) \in E_{f}, \alpha: I \rightarrow X \text { with } \alpha(1)=x, \\
A((x, \beta), \alpha)(s)= & \left(\alpha(s), \gamma_{s}\right) \\
& \text { for } 0 \leqq s \leqq 1,
\end{aligned}
$$

where $\gamma_{s}: I \rightarrow Y$ is defined by

$$
\gamma_{s}(\tau)=\left\{\begin{array}{lc}
\beta\left(\frac{2 \tau}{1+s}\right) & \text { for } 0 \leqq \tau \leqq \frac{1+s}{2}, \\
f \alpha(2(1-\tau)+s) & \text { for } \frac{1+s}{2} \leqq \tau \leqq 1
\end{array}\right.
$$

Also, we have

LEMMA 2. $\quad E_{f} \stackrel{P f}{\longrightarrow} X \stackrel{f}{\rightarrow} Y$ induces for any space $V$ an exact sequence

$$
\pi\left(V, E_{f}\right) \stackrel{(P f)_{*}}{\rightarrow} \pi(V, X) \stackrel{f^{*}}{\longrightarrow} \pi(V, Y) .
$$

Proof. Consider a family of maps $h_{t}: E_{f} \rightarrow Y$ defined by $h_{t}(x, \beta)=\beta(1-t)$ for $x \in X, 0 \leqq t \leqq 1$. This gives rise to a homotopy between $h_{0}=f \circ P f$ and $h_{1}=0$, and thus $f \circ P f \simeq 0$. 
Conversely, given $g: V \rightarrow X$ such that $f_{*}[g]=0$, we can find a homotopy $k_{t}: V \rightarrow Y$ with $k_{0}=0, k_{1}=f \circ g$. For each point $v \in V$ let $\beta(v)$ be the path in $Y$ given by $\beta(v)(s)=k_{s}(v), 0 \leqq s \leqq 1$. Then if we define

$$
k(v)=(g(v), \beta(v)) \quad \text { for } v \in V,
$$

we obtain a map $k: V \rightarrow E_{f}$ such that $P f \circ k=g$, which proves our assertion.

\section{Mapping sequence}

2.1. Suppose $f: X \rightarrow Y$ is a fibering with fibre $F=f^{-1}\left(y_{0}\right)$ and let $i: F \rightarrow X$ be the injection. Using the lifting function $\lambda$ for $f$ we shall define $\Phi: E_{f} \rightarrow F$, $\Psi: E_{f} \rightarrow F$ by

$$
\begin{array}{ll}
\Phi(x)=\left(x, e_{y_{0}}\right) & \text { for } x \in F, \\
\Psi(x, \beta)=\lambda(x, \beta) & \text { for } x \in X, \beta \in E Y \text { with } f(x)=\beta(1) .
\end{array}
$$

Then $\Psi$ is well-defined, since $f \lambda(x, \beta)=y_{0}$ because of the property (i) of the lifting function $\lambda$ (cf. 1.2).

The following theorem plays a crucial role in our later development.

THEOREM 1. $\Phi$ and $\Psi$ are mutually inverse homotopy equivalences and, in addition, the following diagram is commutative up to homotopy

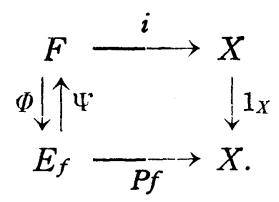

Proof. By definition we have $\Psi \circ \Phi(x)=\lambda\left(x, e_{y_{0}}\right), \Phi \circ \Psi(x, \beta)=\left(\lambda(x, \beta), e_{y_{0}}\right)$. Obviously $\Psi \circ \Phi \simeq 1_{F}$ by the property (ii) of $\lambda$ (cf. 1.2). On the other hand, using the path lifting function $A$ for $f$, a homotopy

$$
((x, \beta), t) \rightarrow\left(\Lambda(x, \beta)(t), \beta_{0, t}\right), \quad(0 \leqq t \leqq 1)
$$

yields $\Phi \circ \Psi \simeq 1_{E_{f}}$. Since $P f \circ \Phi=i$, it follows at once that $i \circ \Psi \simeq P f$, and this concludes the proof.

2.2. We have already seen in Lemma 1 that $P f: E_{f} \rightarrow X$ is a fibering for any map $f: X \rightarrow Y$. This fact enables us to apply Theorem 1 to $P f$ instead of $f$, and we obtain the homotopy commutative diagram 


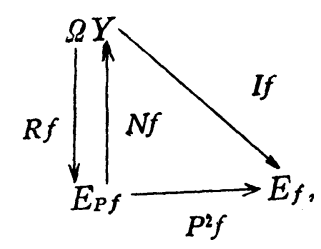

where vertical maps are homotopy equivalences and $P^{n} f(n \geqq 2)$ is defined inductively to be $P\left(P^{n-1} f\right)$. Here we may identify $E_{P f}$ with $\{(\beta, \alpha) \mid \beta \in E Y$, $\alpha \in E X, \beta(1)=f \alpha(1)\}$. Then $R f$ and $N f$ are determined by

$$
\begin{array}{ll}
R f(\beta)=\left(\beta, e_{x_{0}}\right) & \text { for } \beta \in \Omega Y, \\
N f(\beta, \alpha)=\beta \cdot(f \alpha)^{-1} & \text { for }(\beta, \alpha) \in E_{P f},
\end{array}
$$

and, moreover, $P^{2} f(\beta, \alpha)=(\alpha(1), \beta)$ for $(\beta, \alpha) \in E_{P f}$.

Replacing $f$ by $P f$ in the triangle (1), we get the following diagram

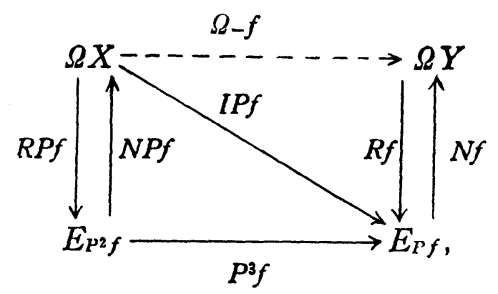

where $\Omega_{-} f: \Omega X \rightarrow \Omega Y$ is the map given by $\Omega_{-} f(\alpha)=(f \alpha)^{-1}$ for $\alpha \in \Omega X$. Since we have $(N f) \circ I P f(\alpha)=N f\left(e_{y_{0}}, \alpha\right)=e_{y_{0}} \cdot(f \alpha)^{-1}$ for $\alpha \in \Omega X$, homotopycommutativity holds in (2).

Let $\sigma_{X}: \Omega X \rightarrow \Omega X$ and $\sigma_{Y}: \Omega Y \rightarrow \Omega Y$ be involutions given by inversions of loops. We set $R_{-} f=R f \circ \sigma_{Y}, R_{-} P f=R P f \circ \sigma_{X}$. Then we have homotopycommutative diagrams

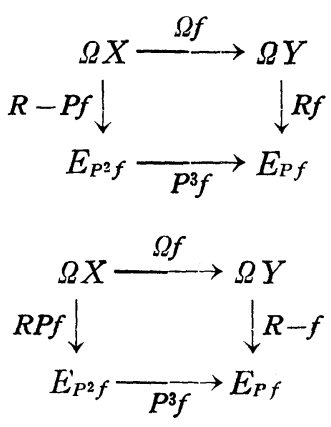

Consider the following diagram 


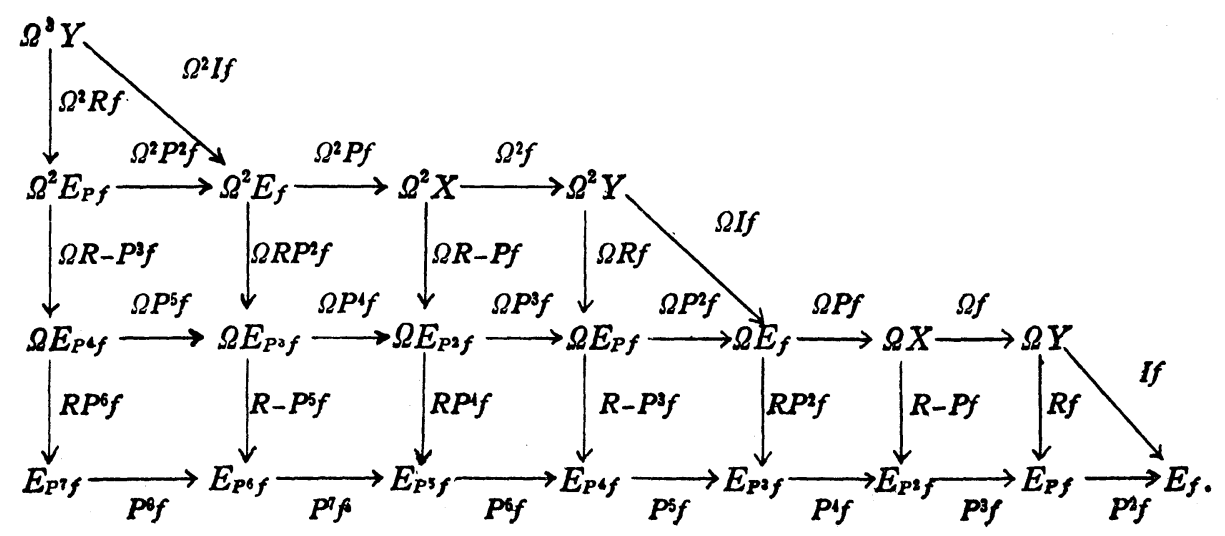

On the lowest ladder homotopy commutativity follows from (1), (3) and (4), as is readily seen. We note that other ladders are obtained from the lowest one by applying the loop functor. Every vertical map is a homotopy equivalence. For brevity we write

$$
R_{n} f= \begin{cases}R P^{n} f & \text { for even } n, \\ R-P^{n} f & \text { for odd } n .\end{cases}
$$

Then we see immediately that vertical equivalences are given by

$$
\begin{aligned}
& R_{3 n-3} f \circ \cdots \circ \Omega^{n-2} R_{3} f \circ \Omega^{n-1} R f: \Omega^{n} Y \equiv E_{P^{3 n-2} f}, \\
& R_{3 n-2} f \circ \cdots \circ \Omega^{n-2} R_{4} f \circ \Omega^{n-1} R_{1} f: \Omega^{n} X \equiv E_{P^{3 n-1} f}, \\
& R_{3 n-1} f \circ \cdots \circ \Omega^{n-2} R_{5} f \circ \Omega^{n-1} R_{2} f: \Omega^{n} E_{f} \equiv E_{P^{3 n f} f}
\end{aligned}
$$

The results obtained above are summarized as follows.

THEOREM 2. The sequence

$$
\begin{aligned}
\mathfrak{M}_{f}: \cdots \rightarrow \Omega^{2} E_{f} \stackrel{\Omega^{2} P f}{\longrightarrow} \Omega^{2} X & \stackrel{\Omega^{2} f}{\longrightarrow} \Omega^{2} Y \stackrel{\Omega I f}{\longrightarrow} \Omega E_{f} \\
& \stackrel{\Omega P f}{\longrightarrow} \Omega X \stackrel{\Omega f}{\longrightarrow} \Omega Y \stackrel{\text { If }}{\longrightarrow} E_{f} \stackrel{P f}{\longrightarrow} X \stackrel{f}{\longrightarrow} Y
\end{aligned}
$$

is homotopically equivalent to the sequence obtained by iterated construction:

$$
\begin{aligned}
& \mathfrak{P} f: \cdots \stackrel{P^{8} f}{\longrightarrow} E_{P^{6} f} \stackrel{P^{7} f}{\longrightarrow} E_{\mathrm{F}^{5} f} \stackrel{P^{6} f}{\longrightarrow} E_{P^{4} f} \stackrel{P^{5} f}{\longrightarrow} E_{P^{3} f} \\
& \qquad \stackrel{P^{4} f}{\longrightarrow} E_{P^{2} f} \stackrel{P^{3} f}{\longrightarrow} E_{P^{f}} \stackrel{P^{2} f}{\longrightarrow} E_{f} \stackrel{P f}{\longrightarrow} X \stackrel{f}{\longrightarrow} Y .
\end{aligned}
$$

We refer to the sequence $\mathfrak{M} f$ above as the mapping sequence of $f$.

Combining Th. 2 with Lemma 2 gives rise to the following 
Corollary 2.1. The mapping sequence $\mathfrak{M} f$ induces for any space $V$ an exact sequence

$$
\begin{aligned}
(\mathfrak{R} f)_{*}: \cdots \rightarrow \pi\left(V, \Omega E_{f}\right) & \stackrel{(\Omega P f)_{*}}{\longrightarrow} \pi(V, \Omega X) \stackrel{(\Omega f)_{*}}{\longrightarrow} \pi(V, \Omega Y) \\
& \stackrel{(I f)_{*}}{\longrightarrow} \pi\left(V, E_{f}\right) \stackrel{(P f)_{*}}{\longrightarrow} \pi(V, X) \stackrel{f_{*}}{\longrightarrow} \pi(V, Y) .
\end{aligned}
$$

Corollary 2.2. If $f: X \rightarrow Y$ is a fibering with fibre $F$, and if $i: F \rightarrow X$, the injection, has a left homotopy inverse (e.g. $F$ is a retract of $X$ ), then the fibering $\Omega f: \Omega X \rightarrow \Omega Y$ admits a cross-section.

Proof. By the assumption above we have $0=\operatorname{Ker}(P f)_{*}=\operatorname{Im}(I f)_{*}$, since $i \equiv P f$ by Th. 1 . Thus $(\Omega f)_{*}$ is onto and hence $\Omega f$ has a right homotopy inverse which may be modified into a cross-section.

\section{Relative mapping sequence}

3.1. It would be natural to expect that the mapping sequence may be relativized.

Let $X \stackrel{h}{\longrightarrow} Y \stackrel{g}{\longrightarrow} Z$ be a triple with $f=g \circ h$ Consider the diagram

$$
\begin{aligned}
& \cdots \rightarrow \Omega^{2} E_{g} \stackrel{\Omega d}{\longrightarrow} \Omega E_{h} \stackrel{\Omega l}{\longrightarrow} \Omega E_{f} \stackrel{\Omega k}{\longrightarrow} \Omega E_{g} \stackrel{d}{\longrightarrow} E_{h} \stackrel{l}{\longrightarrow} E_{f} \stackrel{k}{\longrightarrow} E_{g}
\end{aligned}
$$

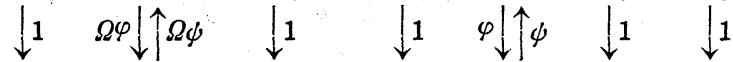

$$
\begin{aligned}
& \cdots \rightarrow \Omega^{2} E_{g} \underset{\Omega I k}{\longrightarrow} \Omega E_{k} \underset{\Omega P k}{\longrightarrow} \Omega E_{f} \underset{\Omega k}{\longrightarrow} \Omega E_{g} \underset{I k}{\longrightarrow} E_{k} \underset{P k}{\rightarrow} E_{f} \underset{k}{\longrightarrow} E_{g} \text {, }
\end{aligned}
$$

where the maps are set as follows:

$$
\begin{array}{ll}
k(x, \beta)=(h(x), \beta) & \text { for } x \in X, \beta \in E Z \text { with } \beta(1)=f(x), \\
l(x, \alpha)=(x, g \alpha) & \text { for } x \in X, \alpha \in E Y \text { with } \alpha(1)=h(x), \\
d(\gamma, \widetilde{\beta})=(x, \gamma) & \text { for } \gamma \in \Omega Y, \widetilde{\beta} \in \Omega E Z \text { with } g \gamma(t)=\widetilde{\beta}(1, t) \\
\psi\left((x, \beta),\left(\alpha, \alpha^{\prime}\right)\right)=(x, \alpha) & \text { for }(x, \beta) \in E_{f},\left(\alpha, \alpha^{\prime}\right) \in E E_{g} \text { with } \\
& h(x)=\alpha(1), \beta(s)=\alpha^{\prime}(s, 1), \\
\varphi(x, \alpha)=\left((x, g \alpha),\left(\alpha, \alpha^{\prime \prime}\right)\right) & \text { for } x \in X, \alpha \in E Y \text { with } \alpha(1)=h(x),
\end{array}
$$

in which $\alpha^{\prime \prime} \in E E Z$ is given by

$$
\alpha^{\prime \prime}(s, t)= \begin{cases}g \alpha(t) & \text { for } s \geqq t, \\ g \alpha(s) & \text { for } s \leqq t\end{cases}
$$

It follows at once from these that $P k \circ \varphi=l, \psi \circ \varphi=1, \phi \circ I k=d$. 
Next, we shall show that $\varphi \circ \psi$ is homotopic to $1: E_{k} \rightarrow E_{k}$. The above definitions lead to

$$
\begin{aligned}
& \varphi \circ \psi\left((x, \beta),\left(\alpha, \alpha^{\prime}\right)\right)=\left((x, g \alpha),\left(\alpha, \alpha^{\prime \prime}\right)\right) \text { for } \\
& h(x)=\alpha(1), \beta(s)=\alpha^{\prime}(s, 1), g \alpha(t)=\alpha^{\prime}(1, t) .
\end{aligned}
$$

Define a family of maps $\gamma_{\tau}: I^{2} \rightarrow Z(0 \leqq \tau \leqq 1)$ by the rule

$$
\gamma_{\tau}(s, t)= \begin{cases}\alpha^{\prime}(\min (1, s+2 \tau-2 \tau t), t) & \text { for } s \geqq t, \\ \alpha^{\prime}(s, t-2 \tau+2 \tau s) & \text { for } s \leqq t, t-2 \tau+2 \tau s \geqq s, \\ \alpha^{\prime}(\min (1,2 s-t+2 \tau-2 \tau s), s) & \text { for } s \leqq t, t-2 \tau+2 \tau s \leqq s,\end{cases}
$$

and define $\beta_{\tau} \in E Z$ by setting $\beta_{\tau}(s)=\gamma_{\tau}(s, 1)$. Then the homotopy given by

$$
\left((x, \beta),\left(\alpha, \alpha^{\prime}\right)\right) \rightarrow\left(\left(x, \beta_{\tau}\right),\left(\alpha, \gamma_{\tau}\right)\right) \in E_{k}, \quad(0 \leqq \tau \leqq 1),
$$

coincides with $1: E_{k} \rightarrow E_{k}$ for $\tau=0$ and with $\varphi \circ \psi$ for $\tau=1$, which proves that $\varphi$ is a homotopy equivalence with $\psi$ as a homotopy inverse, and therefore the diagram above is commutative up to homotopy.

Now we state our results as follows.

TheOREM 3. The sequence constructed for any triple $f=g \circ h$

$$
\rightarrow \Omega^{2} E_{g} \stackrel{\Omega d}{\longrightarrow} \Omega E_{h} \stackrel{\Omega l}{\longrightarrow} \Omega E_{f} \stackrel{\Omega k}{\longrightarrow} \Omega E_{g} \stackrel{d}{\longrightarrow} E_{h} \stackrel{l}{\longrightarrow} E_{f} \stackrel{k}{\longrightarrow} E_{g}
$$

is homotopically equivalent to the "absolute" mapping sequence $\mathfrak{M} k$ of $k: E_{f} \rightarrow E_{g}$ and thus induces for any space $V$ an exact sequence

$$
\cdots \rightarrow \pi\left(V, \Omega E_{g}\right) \stackrel{d_{*}}{\longrightarrow} \pi\left(V, E_{h}\right) \stackrel{l^{*}}{\longrightarrow} \pi\left(V, E_{f}\right) \stackrel{k_{*}}{\rightarrow} \pi\left(V, E_{g}\right) .
$$

The sequence (5) above is said to be the relative mapping sequence of a triple $f=g \circ h$. Note that when $Z$ consists of a single point the sequence (5) is reduced to $\mathfrak{M} h$.

3.2. As an illustration we shall derive some exact sequences found in [9]. Suppose $(X ; A, B)$ be a triad. Let $\Delta: X \rightarrow X \times X$ be the diagonal map, and let $\Delta^{\prime}: \mathrm{A} \cap B \rightarrow A \times B$ be the map determined by $\Delta$. We denote by $\pi$ the projection $A \times B \rightarrow A$. Then the composite $\pi \circ \Delta^{\prime}$ is the injection $i: A \cap B \rightarrow A$. Let $j: E_{\Delta^{\prime}} \rightarrow E_{\Delta}$ be the inclusion.

W. S. Massey [9] defined $\pi_{n}(A / B)$ to be the set of all homotopy classes of

4) Note that this corresponds to a sequence in [6], Prop. 2.3. 
maps $\left(S^{n} ; E_{+}^{n}, E_{-}^{n}\right) \rightarrow(A \cup B ; A, B)$, where $E_{+}^{n}, E_{-}^{n}$ are northern and southern hemispheres respectively. As is easily seen, the following natural isomorphisms hold :

$$
\begin{aligned}
\pi_{n+1}(X ; A, B) & \approx \pi\left(S^{n-1}, E_{j}\right), \\
\pi_{n}(A / B) & \approx \pi\left(S^{n-1}, E_{\Delta^{\prime}}\right), \\
\pi_{n}(X) & \approx \pi\left(S^{n-1}, E_{\Delta}\right), \\
\pi_{n-1}(B) & \approx \pi\left(S^{n-1}, E_{\pi}\right) .
\end{aligned}
$$

Hence we have exact sequences

$$
\begin{aligned}
&(\mathfrak{M} j)_{*}: \cdots \rightarrow \pi_{n+1}(A / B) \rightarrow \pi_{n+1}(X) \\
& \rightarrow \pi_{n+1}(X ; A, B) \rightarrow \pi_{n}(A / B) \rightarrow \pi_{n}(X), \\
&\left(\mathfrak{M} \Delta^{\prime}\right)_{*}: \cdots \rightarrow \pi_{n}(A)+\pi_{n}(B) \rightarrow \pi_{n}(A / B) \\
& \rightarrow \pi_{n-1}(A \cap B) \rightarrow \pi_{n-1}(A)+\pi_{n-1}(B), \\
& \cdots \rightarrow \pi_{n+1}(A, A \cap B) \rightarrow \pi_{n}(B) \rightarrow \pi_{n}(A / B) \\
& \rightarrow \pi_{n}(A, A \cap B) \rightarrow \pi_{n-1}(B),
\end{aligned}
$$

where the last sequence is obtained by applying Th. 3 to the triple $i=\pi \circ \Delta^{\prime}$.

\section{Invariance theorem}

4.1. Suppose we are given the diagram

(6)

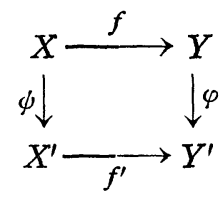

which is commutative up to homotopy. Such a pair of maps $(\varphi, \psi)$ will be called a transformation of $f$ to $f^{\prime}$. With this transformation, together with a fixed homotopy $\Phi$ such that $\Phi_{0}=\varphi \circ f$ and $\Phi_{1}=f^{\prime} \circ \psi$, we associate a map

$$
\chi_{0}=E(\varphi, \psi ; \emptyset): E_{f} \rightarrow E_{f}
$$

by the rule

$$
\chi_{0}(x, \beta)=\left(\psi(x), \beta^{\prime}\right) \text { for } x \in X, \beta \in E Y \text { with } f(x)=\beta(1),
$$

where $\beta^{\prime} \in E Y^{\prime}$ is given by

$$
\beta^{\prime}(s)= \begin{cases}(\varphi \beta)(2 s) & \text { for } 0 \leqq s \leqq \frac{1}{2} \\ \Phi_{2 s-1}(x) & \text { for } \frac{1}{2} \leqq s \leqq 1\end{cases}
$$


In case (6) is strictly commutative, i.e. $\Phi$ can be chosen to be constant, $E(\varphi$, $\psi ; \Phi)$ is denoted simply by $E(\varphi, \psi)$.

Then commutativity holds in the diagram

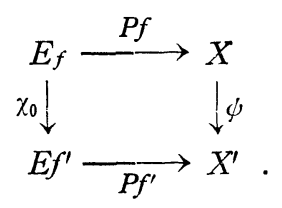

Thus, by proceeding in such a manner, we obtain a transformation $\left(\cdots, \%_{2}\right.$, $\left.\chi_{1}, \chi_{0}, \psi, \varphi\right): \mathfrak{P} f \rightarrow \mathfrak{P} f^{\prime}$, where $\chi_{n}=E\left(\chi_{n-2}, \chi_{n-1}\right), n \geqq 2$ and $\chi_{1}=E\left(\psi, \chi_{0}\right)$. On the other hand, simple calculation shows that $\chi_{0} \circ I f \simeq I f^{\prime} \circ \Omega \varphi$. Hence this yields a transformation $(\cdots, \Omega \psi, \Omega \varphi, \%, \psi, \varphi): \mathfrak{M} f \rightarrow \mathfrak{M} f^{\prime}$. These two transformations are related to each other, as stated in

Lemma 3. In the transformations above, $\mathfrak{M} f \rightarrow \mathfrak{M} f^{\prime}$ and $\mathfrak{P} f \rightarrow \mathfrak{P} f^{\prime}$, the corresponding maps are homotopically equivalent to each other; more precisely, homotopy equivalences obtained in 2.2 yield $\Omega^{n} \chi_{0} \equiv \chi_{3 n}, \Omega^{n} \psi \equiv \chi_{3 n-1}, \Omega^{n} \varphi \equiv \chi_{3 n-2}$.

4.2. Let $(\varphi, \psi)$ be as before, and let $\left(\varphi^{\prime}, \psi^{\prime}\right)$ be a transformation of $f^{\prime}$ to $f^{\prime \prime}$ with a fixed homotopy $\Phi^{\prime}$ such that $\Phi_{0}^{\prime}=\varphi^{\prime} \circ f^{\prime}$ and $\Phi_{1}^{\prime}=f^{\prime \prime} \circ \psi^{\prime}$. We define a homotopy $\left(\Phi^{\prime} \circ \Phi\right): \varphi^{\prime} \circ \varphi \circ f \simeq f^{\prime \prime} \circ \psi^{\prime} \circ \psi$ by setting

$$
\left(\Phi^{\prime} \circ \mathscr{D}\right)_{t}= \begin{cases}\varphi^{\prime} \circ \Phi_{2 t} & \text { for } 0 \leqq t \leqq \frac{1}{2}, \\ \Phi_{2 t-1}^{\prime} \circ \psi & \text { for } \frac{1}{2} \leqq t \leqq 1 .\end{cases}
$$

Then it is readily verified that the following homotopy holds :

LEMMA 4. $E\left(\varphi^{\prime} \circ \varphi, \phi^{\prime} \circ \psi ;\left(\Phi^{\prime} \circ \Phi\right)\right) \simeq E\left(\varphi^{\prime}, \phi^{\prime} ; \Phi^{\prime}\right) \circ E(\varphi, \psi ; \emptyset)$.

Lemma 5. Let $(\varphi, \psi)$ be a transformation of $f$ to $f^{\prime}$ with a fixed homotopy $\Phi$, and let $\varphi \simeq \bar{\varphi}, \psi \simeq \bar{\psi}$. Then there exists a homotopy $\bar{\Phi}: \bar{\varphi} \circ f \simeq f^{\prime} \circ \bar{\psi}$ such that $E(\bar{\varphi}, \bar{\psi} ; \bar{\Phi}) \simeq E(\varphi, \psi ; \Phi)$.

The proof of Lemma 5 may be proceeded in the same manner as in [12], $2.5, \mathrm{~B})$ and thus is omitted.

These two Lemmas yield the next result.

Lemma 6. Let $(\varphi, \psi)$ be a transformation of $f$ to $f^{\prime}$ with a fixed homotopy Ф. If $\varphi$ and $\psi$ are homotopy equivalences, so is $\psi_{0}=E(\varphi, \psi ; \emptyset): E_{f} \rightarrow E_{f}$. 
From this we can derive a invariance theorem for mapping sequences.

THEOREM 4. If in the transformation $\mathfrak{M} f \rightarrow \mathfrak{M} f^{\prime}$ or $\mathfrak{P} f \rightarrow \mathfrak{P} f^{\prime}$ two consecutive maps are homotopy equivalences, so are the following ones. In particular, if $f \equiv f^{\prime}$, then each map in this transformation is a homotopy equivalence.

Corollary 4.1. (Invariance of mapping sequence) Let $f, f^{\prime}: X \rightarrow Y$ be homotopic. Then $\mathfrak{M} f$ and $\mathfrak{P} f$ are homotopically equivalent to $\mathfrak{M} f^{\prime}$ and $\mathfrak{P} f^{\prime}$ respectively.

Corollary 4.2. If $f: X \rightarrow Y$ is nullhomotopic, then $\mathfrak{M} f$ coincides up to homotopy equivalences with the next sequence

$$
\cdots \rightarrow \Omega X \times \Omega^{2} Y \stackrel{\Omega \pi}{\longrightarrow} \Omega X \stackrel{0}{\longrightarrow} \Omega Y \stackrel{i}{\longrightarrow} X \times \Omega Y \stackrel{\pi}{\longrightarrow} X \stackrel{0}{\longrightarrow} Y,
$$

where $\pi$ and $i$ denote the projection and injection respectively; especially, if $f$ : $X \rightarrow Y$ is an inessential fibering with fibre $F$, then $F \equiv X \times \Omega Y$.

CoRollaRY 4.3. If $f: X \rightarrow Y$ is homotopically equivalent to $\Omega f^{\prime}: \Omega X^{\prime} \rightarrow \Omega Y^{\prime}$ for some $f^{\prime}: X^{\prime} \rightarrow Y^{\prime}$, then $E_{f} \equiv \Omega E_{f}$.

Proof. It follows from Th. 2 that $f \equiv \Omega f^{\prime} \equiv P^{3} f^{\prime}$. Hence the above Lemma 6 gives homotopy equivalence $E_{f} \equiv E_{P^{3} f^{\prime}}$. Thus, again in view of Th. 2, we have the desired conclusion $E_{f} \equiv \Omega E_{f^{\prime}}$, since $E_{P^{3} f^{\prime}} \equiv \Omega E_{f^{\prime}}$.

\section{Applications to homotopy equivalences}

5.1. The following relations between $f: X \rightarrow Y$ and $f_{*}: \pi(V, X) \rightarrow \pi(V$, $Y)$ are used freely.

(i) $f$ is nullhomotopic if and only if $f_{*}$ vanishes for every space $V$.

(ii) $f$ has a right homotopy inverse if and only if $f_{*}$ is onto for each $V$.

(iii) If $f$ has a left homotopy inverse, then $\operatorname{Ker} f_{*}=0$ for any $V$.

(iv) (a partial converse to (iii)). If $f_{*}$ has kernel zero for every $V$, then $\Omega f$ admits a left homotopy inverse.

Proof of $(i v)$. Our hypothesis, together with the exactness of $(\mathfrak{M} f)_{*}$, implies that $P f \simeq 0$. Accordingly, Cor. 4.2 asserts that $M P f$ is equivalent to the sequence

$$
\rightarrow \Omega E_{f} \stackrel{0}{\longrightarrow} \Omega X \stackrel{i}{\longrightarrow} E_{f} \times \Omega X \stackrel{\pi}{\longrightarrow} E_{f} \stackrel{0}{\longrightarrow} X,
$$


where $i$ and $\pi$ are the injection and projection respectively. Hence we see from Th. 2 that $i \equiv I P f \equiv P^{2} P f=P^{3} f \equiv \Omega f$. This shows that $\Omega f$, like $i$, has a left homotopy inverse.

The mapping sequence provides a useful tool for establishing various homotopy equivalences, as will be shown in what follows.

5.2. Let us consider the injection $i: X \vee Y \rightarrow X \times Y$, whose mapping sequence is written

$$
\cdots \rightarrow \Omega(X \vee Y) \stackrel{\Omega i}{\longrightarrow} \Omega(X \times Y)=\Omega X \times \Omega Y \stackrel{I i}{\longrightarrow} E_{i} \stackrel{P i}{\longrightarrow} X \vee Y \stackrel{i}{\longrightarrow} X \times Y .
$$

First of all, we note

LEMMA $7 . \quad I i \simeq 0$.

Proof. Let us introduce the following subspaces of $\Omega X$ and $\Omega Y$ :

$$
\begin{aligned}
& \Omega X=\left\{\alpha \in \Omega X \mid \alpha\left(\left[0, \frac{1}{2}\right]\right)=x_{0}\right\}, \\
& \Omega Y=\left\{\beta \in \Omega Y \mid \beta\left(\left[\frac{1}{2}, 1\right]\right)=y_{0}\right\} .
\end{aligned}
$$

Then injections $\bar{\Omega} X \rightarrow \Omega X$ and $\underline{\Omega} Y \rightarrow \Omega Y$ are evidently homotopy equivalences with homotopy inverses $\alpha \rightarrow e_{x_{0}} \cdot \alpha$ and $\beta \rightarrow \beta \cdot e_{y_{0}}$ respectively. Consider now the commutative diagram

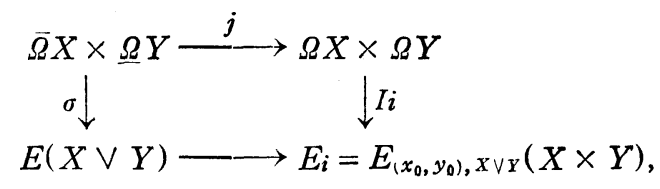

where $\sigma$ is given by $\sigma(\alpha, \beta)=\alpha \times \beta$, the product path of $\alpha$ and $\beta$, and the other maps are all injections. Since $E(X \vee Y)$ is contractible, it follows from commutativity that $I i \circ j \simeq 0$. $j$, as the product of homotopy equivalences, is a homotopy equivalence. Therefore, it follows $I i \simeq 0$, as we wish to prove.

Combining this lemma with Cor. 4.2 we obtain an equivalence $E_{t i} \equiv \Omega X \times$ $\Omega Y \times \Omega E_{i}$, while by virtue of Th. 2 we see that $I i \equiv P^{2} i, E_{P^{2} i} \equiv \Omega(X \vee Y)$. Combining these results we conclude

Proposition 1. $\Omega(X \vee Y) \equiv \Omega X \times \Omega Y \times \Omega E_{\left(x_{0}, y_{0}\right), X \vee Y}(X \times Y)$.

This may be regarded as a result dual to Hilton formula ([12], Th. 15). 
5.3. The following is, in a sense, a substitute for Sugawara's Lemma ([16], p. 118) in case $X$ is not a CW-complex.

Proposition 2. Let $X$ be an $H$-space with $H$-structure $\mu: X \times X \rightarrow X$ and let $f, g: X \times X \rightarrow X$ be defined by

$$
\begin{aligned}
& f\left(x_{1}, x_{2}\right)=\left(\mu\left(x_{1}, x_{2}\right), x_{1}\right) \\
& g\left(x_{1}, x_{2}\right)=\left(\mu\left(x_{1}, x_{2}\right), x_{2}\right) \\
& \text { for } x_{1}, x_{2} \in X \text {. }
\end{aligned}
$$

Then $\Omega f$ and $\Omega g$ are both homotopy equivalences. In particular, if $X$ is a $C W$ complex such that $X \times X$ is also a $C W$-complex, then $f$ and $g$ are homotopy equivalences.

Proof. Let $\pi_{1}, \pi_{2}: X \times X \rightarrow X$ be projections onto the first and second factors respectively. Since $\pi_{2} \circ f=\pi_{1}$, Th. 3 yields the sequence

$$
\cdots \rightarrow \Omega E_{\pi_{1}} \stackrel{\Omega k}{\longrightarrow} \Omega E_{\pi_{2}} \longrightarrow E_{f} \longrightarrow E_{\pi_{1}} \stackrel{k}{\longrightarrow} E_{\pi_{2}}
$$

where $k$ is given by $k\left(\left(x_{1}, x_{2}\right), \alpha\right)=\left(\left(\mu\left(x_{1}, x_{2}\right), x_{1}\right), \alpha\right)$ for $x_{1}, x_{2} \in X, \alpha \in E X$ with $\alpha(1)=x_{1}$.

Let $k^{\prime}: E_{\pi_{1}} \rightarrow E_{\pi_{2}}$ be defined by $k^{\prime}\left(\left(x_{1}, x_{2}\right), \alpha\right)=\left(\left(\mu\left(x_{0}, x_{2}\right), x_{0}\right), e_{x_{0}}\right)$. Then $k$ is homotopic to $k^{\prime}$ via a homotopy defined by

$$
\left(\left(x_{1}, x_{2}\right), \alpha\right) \rightarrow\left(\left(\mu\left(\alpha(1-t), x_{2}\right), \alpha(1-t)\right), \alpha_{0,1-t}\right), 0 \leqq t \leqq 1 .
$$

Consider now the following commutative diagram

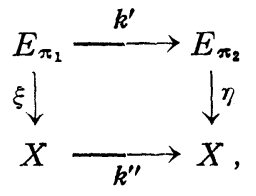

where the maps are set as follows,

$$
\begin{array}{rlrl}
\xi\left(\left(x_{1}, x_{2}\right), \alpha\right) & =x_{2}, & \text { for } x_{1}, x_{2} \in X, \alpha, \beta \in E X \text { with } \\
& \alpha(1)=x_{1}, \beta(1)=x_{2}, \\
\eta\left(\left(x_{1}, x_{2}\right), \beta\right) & =x_{1}, & \\
k^{\prime \prime}(x) & =\mu\left(x_{0}, x\right) & \text { for } x \in X .
\end{array}
$$

We see that $\xi$ and $\eta$ are both homotopy equivalences and that $k^{\prime \prime}$ is homotopic to $1_{X}$ by the condition imposed upon $H$-structure, so that $k$ and $\Omega k$ are homotopy equivalences. It follows from exactness of the sequence induced by 
(7) that $\pi\left(V, E_{f}\right)=0$ for each space $V$. This implies that $E_{f}$ is contractible to a point. Upon examination of $\mathfrak{M} f$ one sees that $\Omega f$ is a homotopy equivalence; similarly for $g$.

The latter statement of the proposition follows at once by application of a well-known theorem of J.H.C. Whitehead [17] to the first assertion.

It is readily verified that if the above map $f: X \times X \rightarrow X$ has a right homotopy inverse, then $X$ admits a right homotopy inversion. Thus the following is an immediate conclusion.

Corollary. (Sugawara [16]) Let $X$ be an H-space which is a CW-complex and let $X \times X$ be also a $C W$-complex. Then $X$ has right and left homotopy inversions.

5.4. In the sequel we shall establish various equivalences related to fiberings. To this end we need the following lemma which is dual to Lemma 8 of D. Puppe ([12], 3.4).

Lemma 8. Let $f: X \rightarrow Y$ be any map such that Pf: $E_{f} \rightarrow X$ admits a left homotopy inverse $l: X \rightarrow E_{f}$. Let $\psi: X \rightarrow Y \times E_{f}$ be determined by $f$ and $l$, i.e. $\psi(x)=(f(x), l(x))$ for $x \in X$. Then $\Omega \psi: \Omega X \rightarrow \Omega Y \times \Omega E_{f}$ is a homotopy equivalence.

Proof. It is clear from the definition of $\psi$ that the square

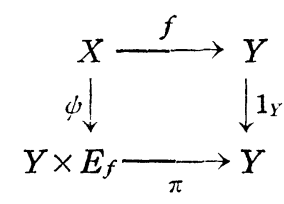

is commutative. Here $\pi$ denotes the projection on the first factor. Thus it induces a transformation of $\mathfrak{M} f$ to $\mathfrak{M} \pi$. Observe that the projection $\rho: E_{\pi}=$ $E_{f} \times E Y \rightarrow E_{f}$ is a homotopy equivalence since $E Y$ is contractible.

Consider the map

$$
\chi_{0}=E\left(1_{Y}, \psi\right): E_{f} \rightarrow E_{\pi}=E_{f} \times E Y,
$$

whose definition leads to the following calculation 


$$
\begin{aligned}
\left(\rho \circ \chi_{0}\right)(x, \beta) & =\rho\left(\psi(x), \beta \cdot e_{f(x)}\right) \quad \text { for } x \in X, \beta \in E Y \text { with } \\
& f(x)=\beta(1), \\
& =\rho\left(l(x), \beta \cdot e_{f(x)}\right) \\
& =l(x) \\
& =l \circ P f(x, \beta) .
\end{aligned}
$$

Therefore, our assumption implies $\rho \circ \chi_{0} \simeq 1_{E f}$. It follows from the previous remark that $\chi_{0}$ is a homotopy equivalence. Hence the transformation $\mathfrak{M} f \rightarrow \mathfrak{M}_{\pi}$ contains two consecutive maps $\chi_{0}$ and $\Omega 1_{Y}=1_{\Omega Y}$ both of which are homotopy equivalences. Thus, in view of Th. 4, we see that $\Omega \psi: \Omega X \rightarrow \Omega\left(Y \times E_{f}\right)$ is also a homotopy equivalence. This completes the proof.

Combining this Lemma with Th. 1 then gives

Proposition 3. (cf. I.M. James and J.H.C. Whitehead [8]) Let $f: X \rightarrow Y$ be a fibering with fibre $F$, and let $F$ be a retract of $X$. Let $r: X \rightarrow F$ be a retraction. Then the map $\psi: X \rightarrow Y \times F$ defined by $\psi(x)=(f(x), r(x))$ induces a homotopy equivalence $\Omega \psi: \Omega X \equiv \Omega(Y \times F)$. In particular, if $X$ and $Y$ are pathwise connected spaces dominated by $\mathrm{CW}$-complexes, then $\psi$ is a homotopy equivalence.

Proposition 4. Let $f: X \rightarrow Y$ be a fibering which admits a cross-section, and let $F$ be its fibre. Then $\Omega^{2} X \equiv \Omega^{2}(Y \times F)$.

Proof. Since $\Omega f: \Omega X \rightarrow \Omega Y$ also admits a cross-section, the exactness of $(\mathfrak{M} f)_{*}$ implies $\operatorname{Ker}(P f)_{*}=0$. By virtue of (iv) in 5.1, it results that $\Omega P f$ admits a left homotopy inverse. Since $\Omega P f \equiv P^{3} P f=P^{4} f=P\left(P^{3} f\right)$ by Th. 2 , we can apply Lemma 8 to $P^{3} f: E_{P^{2} f} \rightarrow E_{P f}$. Then we have $\Omega E_{P^{2} f} \equiv \Omega\left(E_{P f} \times\right.$ $\left.E_{P^{3} f}\right)$. We note that $E_{P^{3} f} \equiv \Omega E_{f} \equiv \Omega F, E_{P^{2} f} \equiv \Omega X, E_{P f} \equiv \Omega Y$ on account of Th. 1 and 2. We thus see that $\Omega^{2} X \equiv \Omega^{2}(Y \times F)$.

Following Peterson and Thomas [11], we shall say that a fibering $f: X \rightarrow Y$ with fibre $F$ is principal if there exist maps

$$
\mu: F \times X \rightarrow X, \quad h:\left\{\left(x_{1}, x_{2}\right) \in X \times X \mid f\left(x_{1}\right)=f\left(x_{2}\right)\right\} \rightarrow F
$$

satisfying the conditions:

(i) $f \circ \mu\left(x_{1}, x_{2}\right)=f\left(x_{2}\right) \quad$ for $x_{1} \in F, x_{2} \in X$,

(ii) $\mu \mid F \times F$ gives an $H$-structure of $F$,

(iii) $\left(x_{1}, x_{2}\right) \rightarrow \mu\left(h\left(x_{1}, x_{2}\right), x_{1}\right)$ is homotopic to $\left(x_{1}, x_{2}\right) \rightarrow x_{2}$ via a homotopy 
which moves image-points along fibers. ${ }^{5}$

Then we can strengthen Prop. 4 for principal fiberings as follows.

Proposition 5. Suppose that $f: X \rightarrow Y$ is a principal fibering with fibre $F$ and that it admits a cross-section. Then there exists a commutative triangle

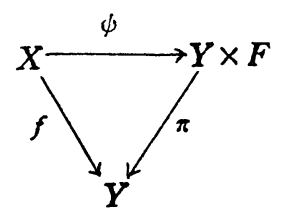

such that $\Omega \psi: \Omega X \equiv \Omega(Y \times F)$, where $\pi$ is the projection.

Proof. We denote by $s: Y \rightarrow X$ a cross-section, and let $\mu, h$ be as above. We now define $\xi: X \rightarrow F$ by

$$
\xi(x)=\mu\left(h(s \circ f(x), x), x_{0}\right) \quad \text { for } x \in X .
$$

Since $\xi(x)=\mu\left(h\left(x_{0}, x\right), x_{0}\right)$ for $x \in F$, it follows from the condition (iii) of principal fiberings that $\xi: X \rightarrow F$ is a left homotopy inverse of the injection $F \rightarrow X$. It follows from Th. 1 that $P f: E_{f} \rightarrow X$ also admits a left homotopy inverse. This fact enables us to apply Lemma 8. Indeed, if we define $\psi$ : $X \rightarrow Y \times F$ by $\phi(x)=(f(x), \xi(x))$, we see that $\Omega \psi$ is a homotopy equivalence, which proves our assertion.

Finally we shall prove

Proposition 6. Let $f: X \rightarrow Y$ be a fibering such that the fibre $F$ is contractible to a point in $X$. Then $\Omega Y \equiv F \times \Omega X$.

Proof. Since $F \equiv E_{f}$ by Th. 1, our assumption implies that $P f: E_{f} \rightarrow X$ is nullhomotopic. Therefore, we see from Cor. 4.2 that $E_{P f} \equiv E_{f} \times \Omega X$. With reference to Th. 1 and 2 we have $E_{f} \equiv F$ and $E_{P f} \equiv \Omega Y$, which lead to the desired conclusion.

Upon examination, one sees easily that homotopy equivalences of Prop. 6

$$
\eta: F \times \Omega X \rightarrow \Omega Y, \quad \kappa: \Omega Y \rightarrow F \times \Omega X
$$

are given, using a contraction $\Phi_{s}: F \rightarrow X$ such that $\Phi_{1}=0$, by

5) This condition is more restrictive than the one given in [11]. 


$$
\eta(x, \alpha)= \begin{cases}f \circ \Phi_{2 s}(x) & \text { for } 0 \leqq s \leqq \frac{1}{2}, \\ f \alpha(2-2 s) & \text { for } \frac{1}{2} \leqq s \leqq 1,\end{cases}
$$

where $\alpha^{\prime} \in \Omega X$ is determined by

$$
\alpha^{\prime}(s)= \begin{cases}\Lambda\left(x_{0}, \beta\right)(1-2 s), & 0 \leqq s \leqq \frac{1}{2}, \\ \Phi_{2 s-1} \lambda\left(x_{0}, \beta\right), & \frac{1}{2} \leqq s \leqq 1\end{cases}
$$

Here we denote a lifting function and path lifting function for $f$ by $\lambda, \Lambda$ respectively.

Corollary (cf. Spanier and J.H.C. Whitehead [15]). Under the same situation as in Proposition 6, the fibre $F$ is an H-space.

Corollary. If $f: X \rightarrow Y$ is a fibering with the contractible total $X$, then $\eta: F \rightarrow \Omega Y$ defined by $[\eta(x)](s)=f \circ \emptyset_{s}(x)$ for $x \in F, 0 \leqq s \leqq 1$ is a (strict) homotopy equivalence, where $\Phi_{s}$ denotes a contraction of $X$.

The latter corollary is a variant of a result due to H. Samelson [13].

\section{Part II. Mapping SEQUences AND SUSPensions}

\section{Preliminaries}

6.1. We start by recalling all the basic definitions and results stated in [12] in so far as they are necessary for the application we have in view.

Given a map $f: X \rightarrow Y$, let $C_{f}$ be the mapping cone of $f$, the space obtained from $C X \cup Y$ by identifying $(x, 1)$ with $f(x)$, where $C X$ denotes the cone over $X$. We denote by $S$ the reduced suspension functor. With these notations, it is known that the sequence

$$
\mathfrak{A} f: X \stackrel{f}{\rightarrow} Y \stackrel{P^{\prime} f}{\rightarrow} C_{f} \stackrel{Q f}{\rightarrow} S X \stackrel{S f}{\rightarrow} S Y \stackrel{S P^{\prime} f}{\rightarrow} S C_{f} \rightarrow \cdots
$$

has the same properties as $\mathfrak{M} f$, where the maps involved are defined in the following fashion: 


$$
\begin{gathered}
P^{\prime} f(y)=y \in Y \subset C_{f} \quad \text { for } y \in Y, \\
Q f(x, s)=(x, s) \quad \text { for }(x, s) \in C X, Q f(y)=\left(x_{0}, 1\right) \\
\text { for } y \in Y .
\end{gathered}
$$

Next, given a transformation from $f: X \rightarrow Y$ to $f^{\prime}: X^{\prime} \rightarrow Y^{\prime}$

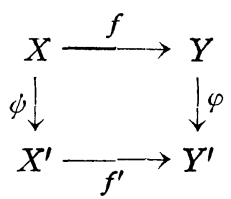

with a fixed homotopy $\Phi$ such that $\Phi_{0}=\varphi \circ f, \Phi_{1}=f^{\prime} \circ \phi$, we construct the map

$$
\chi_{0}^{\prime}=C(\psi, \varphi ; \emptyset): C_{f} \rightarrow C_{f}
$$

by setting

$$
\begin{gathered}
\chi_{0}^{\prime}(y)=\varphi(y) \\
\chi_{0}^{\prime}(x, s)= \begin{cases}(\psi(x), 2 s) & \text { for } y \in Y \subset C_{f}, \\
\Phi_{2-2}(x) & \text { for } x \in X, 0 \leqq s \leqq \frac{1}{2},\end{cases}
\end{gathered}
$$

Then we have (D. Puppe [12], Lemma 7)

Lemмa 9. If $\varphi$ and $\psi$ are both homotopy equivalences, so is $\chi_{0}^{\prime}$.

6.2. Following Eckmann and Hilton [4], we shall say that $f: X \rightarrow Y$ is a cofibering if it has the homotopy lowering property for all spaces, i.e., if, for $g: X \rightarrow Z, G: Y \rightarrow Z$ with $g=G \circ f$, each homotopy of $g$ can be obtained by composing $f$ with some homotopy of $G$. The quotient space $Y / f(X)$ is called the cofibre of $f$. Then we shall prove

Lemma 10. Let $f: X \rightarrow Y$ be a map and let $M_{f}$ be its mapping cylinder. In order that $f$ be a cofibering, it is necessary and sufficiant that there exist a map $\Lambda^{\prime}: Y \times I \rightarrow M_{f}$ such that $\Lambda^{\prime}(f(x), t)=(x, t), \Lambda^{\prime}(y, 1)=y$ for $x \in X, y \in Y$, $0 \leqq t \leqq 1$.

Proof. Suppose $f$ is a cofibering. We define a map $Y \times 1 \rightarrow M_{f}$ by $(y, 1)$ $\rightarrow y$. Then the homotopy $X \times I \rightarrow M_{f}$ given by $(x, t) \rightarrow(x, t)$ can be lowered to a homotopy $\Lambda^{\prime}: Y \times I \rightarrow M_{f}$ which is a desired function.

Conversely, let $G: Y \rightarrow Z, g_{t}: X \rightarrow Z$ be such that $G(f(x))=g_{1}(x)$ for $x \in X$, where $0 \leqq t \leqq 1$. Using the above $A^{\prime}$, we define a homotopy 


$$
G^{\prime} \circ \Lambda^{\prime}: Y \times I \rightarrow Z,
$$

where $G^{\prime}: M_{f} \rightarrow Z$ is given by taking $G^{\prime}(y)=G(y), G^{\prime}(x, s)=g_{s}(x)$ for $y \in Y$, $x \in X, 0 \leqq s \leqq 1$. This proves the sufficiency.

We see from the above lemma that if $f$ is a cofibering then $f$ is necessarily univalent, so that from now on we consider only inclusion cofiberings. With $A^{\prime}$ above, define $\lambda^{\prime}: Y \rightarrow M_{f}$ by

$$
\lambda^{\prime}(y)=\Lambda^{\prime}(y, 0) \quad \text { for } y \in Y .
$$

Then this $\lambda^{\prime}$, called a extension function for $f$, has the following properties:

(i) $\lambda^{\prime}(x)=(x, 0) \quad$ for $x \in X$,

(ii) the composition $r \circ \lambda^{\prime}$ with the retraction $r: M_{f} \rightarrow Y$ is homotopic to the identity $1_{Y}$ of $Y$ via a homotopy which sends $X$ into $X$.

Consider the diagram

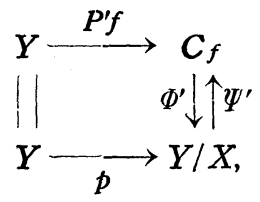

where $\phi$ is the natural projection, $\Phi^{\prime}$ the map $C_{f} \rightarrow C_{f} / C X=Y / X$ obtained by pinching $C X$ to a point and $\Psi^{\prime}$ the map induced by $\lambda^{\prime}: Y \rightarrow M_{f}$. Then we have

LEMma 11. $\Phi^{\prime}$ and $\Psi^{\prime}$ are mutually inverse homotopy equivalences and, furthermore, the above diagram is commutative up to homotopy. (Puppe [12])

Proof. It follows from (ii) that $\Phi^{\prime} \circ \Psi^{\prime} \simeq 1$. On the other hand, the homotopy given by

$$
\begin{aligned}
(x, s) & \rightarrow \Lambda^{\prime}(x, s t), & & \text { for } 0 \leqq t \leqq 1,(x, s) \in X \times I, \\
y & \rightarrow \Lambda^{\prime}(y, t) & & \text { for } 0 \leqq t \leqq 1, \in Y
\end{aligned}
$$

yields a homotopy connecting $\Psi^{\prime} \circ \Phi^{\prime}$ with $1_{c_{f}}$. It is obvious that $p=\Phi^{\prime} \circ P^{\prime} f$, so our assertion is proved.

The following is easily read from the proof of Puppe's Lemma 6 [12].

Lemma 12. $P^{\prime} f: Y \rightarrow C_{f}$ is a cofibering, whose $\lambda^{\prime}$ and $A^{\prime}$ are given by

$$
\lambda^{\prime}(x, s)=\left\{\begin{array}{l}
(x, 2 s) \in C_{f}, 0 \leqq s \leqq \frac{1}{2}, \quad(x, s) \in C X, \\
(f(x), 2-2 s) \in Y \times I, \frac{1}{2} \leqq s \leqq 1,(x, s) \in C X,
\end{array}\right.
$$




$$
\begin{aligned}
\lambda^{\prime}(y) & =(y, 0) \in Y \times I, y \in Y \subset C_{f}, \\
\Lambda^{\prime}((x, s), t) & =\left\{\begin{array}{l}
\left(x, \frac{2 s}{1+t}\right), 0 \leqq s \leqq \frac{1+t}{2} \\
(f(x), 2-2 s+t), \frac{1+t}{2} \leqq s \leqq 1 .
\end{array}\right. \\
\Lambda^{\prime}(y, t) & =(y, t) .
\end{aligned}
$$

Finally we state the following well known lemma which makes it possible to convert any map into a map of simpler type.

Lemma 13 ([3] or [1]). Any map is equivalent to a fibering (or a cofibering).

Proof. Given any map $f: X \rightarrow Y$, let $M_{f}$ be the mapping cylinder of $f$ and we set

$$
Z_{f}=\left\{(x, \beta) \mid x \in X, \beta \in E_{Y, Y}(Y), f(x)=\beta(1)\right\} .
$$

Let $p: Z_{f} \rightarrow Y, i: X \rightarrow M_{f}$ be defined by setting $p(x, \beta)=\beta(0), i(x)=(x, 0)$. Then we see at once that $p$ is a fibering with fibre $E_{f}$ and that $i$ is a cofibering with cofibre $C_{f}$, both of which are clearly equivalent to $f$ respectively.

6.3. Let $f: X \rightarrow Y$ be a map. We shall define left operations (cf. [12], 4.3)

$$
\mu: \Omega Y \times E_{f} \rightarrow E_{f}, \quad \mu^{\prime}: C_{f} \rightarrow S X \vee C_{f}
$$

as follows.

$$
\begin{aligned}
& \mu(\omega,(x, \beta))=(x, \omega \cdot \beta) \quad \text { for } \omega \in \Omega Y, x \in X, \beta \in E Y, \\
& \mu^{\prime}(y)=y \quad \text { for } y \in Y \subset C_{f} \text {, } \\
& \mu^{\prime}(x, s)=\left\{\begin{array}{l}
(x, 2 s) \in S X, s \leqq \frac{1}{2} \\
(x, 2 s-1) \in C X, s \geqq \frac{1}{2}
\end{array} \quad \text { for }(x, s) \in C X \subset C_{f}\right.
\end{aligned}
$$

These induce natural $H$ - and $H^{\prime}$-structures (cf. [4]) $\Omega Y \times \Omega Y \rightarrow \Omega Y, S X \rightarrow S X \vee$ $S X$ which are also denoted by $\mu, \mu^{\prime}$. We have several properties about them. For example,
a) The diagrams 


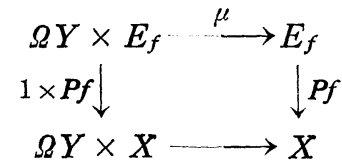

are commutative

b) $\Omega Y \times \Omega Y \times E_{f} \stackrel{1 \times \mu}{\longrightarrow} \Omega Y \times E_{f}$

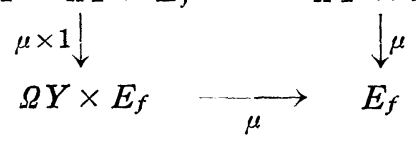

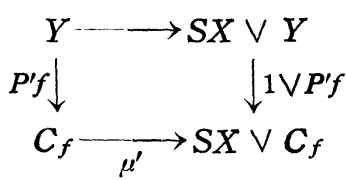

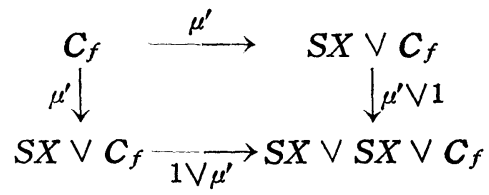

are homotopy-commutative.

Moreover $E_{f}$ has a principal structure as mentioned in 5.4. Let $E_{f}^{*}$ be the reciprocal image of the diagonal under $P f \times P f: E_{f} \times E_{f} \rightarrow X \times X$. If we define $h: E_{f}^{*} \rightarrow \Omega Y$ by taking

$$
h\left((x, \beta),\left(x, \beta^{\prime}\right)\right)=\beta^{\prime} \cdot \beta^{-1},
$$

we obtain a homotopy commutative diagram

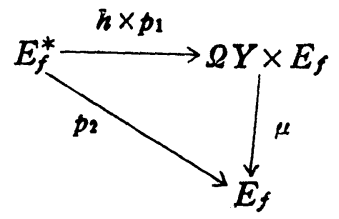

where $p_{i}: E_{f}^{*} \rightarrow E_{f}$ are defined by $p_{i}\left(z_{1}, z_{2}\right)=z_{i}, i=1,2, z_{i} \in E_{f}$. This homotopy $\mu^{\circ}\left(h \times p_{1}\right) \simeq p_{2}$ can be chosen so as to move image-points along fibres.

\section{Connecting diagram ${ }^{6)}$}

7.1. We shall call a triple $X \stackrel{f}{\rightarrow} Y \stackrel{g}{\rightarrow} Z$ nullhomotopic if and only if the composition $g \circ f$ is homotopic to the constant. Given such a triple with a definite homotopy $H_{t}: X \rightarrow Z$ such that $H=g \circ f, H_{1}=0$, we form the connecting diagram of $X \stackrel{f}{\longrightarrow} Y \stackrel{g}{\longrightarrow} Z$ which is written as follows.

$$
\begin{aligned}
& \ldots \stackrel{\Omega f}{\rightarrow} \Omega Y \stackrel{I f}{\longrightarrow} E_{f} \stackrel{P f}{\longrightarrow} X \stackrel{f}{\rightarrow} Y \stackrel{P^{\prime} f}{\longrightarrow} C_{f} \stackrel{Q f}{\longrightarrow} S X \stackrel{S-f}{\rightarrow} S Y \stackrel{S P^{\prime} f}{\longrightarrow} \ldots \\
& \eta_{f, g} \downarrow \quad \xi_{f, g} \downarrow\|\quad\| \xi_{f, g}^{\prime} \quad \downarrow^{\prime} f, 0 \\
& \cdots \underset{\Omega P g}{\longrightarrow} \Omega Y \underset{\Omega-g}{\longrightarrow} \Omega Z \underset{I g}{\longrightarrow} E_{g} \underset{P g}{\longrightarrow} Y \underset{g}{\longrightarrow} Z \underset{P^{\prime} g}{\longrightarrow} C_{g} \underset{Q g}{\longrightarrow} S Y \underset{S g}{\longrightarrow} \cdots
\end{aligned}
$$

6) This construction was inspired from a discussion in [5]. 
in which the maps exhibited are defined by setting

$$
\begin{aligned}
& \xi_{f, g}(x)=(f(x), \gamma), \gamma(s)=H_{1-s}(x) \quad \text { for } x \in X, 0 \leqq s \leqq 1 \text {, } \\
& \eta_{f, g}(x, \beta)= \begin{cases}H_{1-2}(x) & \text { for } 0 \leqq s \leqq \frac{1}{2},(x, \beta) \in E_{f}, \\
g \beta(2-2 s) & \text { for } \frac{1}{2} \leqq s \leqq 1,(x, \beta) \in E_{f},\end{cases} \\
& \hat{\xi}_{f, g}^{\prime}(y)=g(y), \xi_{f, g}^{\prime}(x, s)=H_{1-s}(x) \quad \text { for } y \in Y,(x, s) \in C X \text {, } \\
& \eta_{f, g}^{\prime}(x, s)= \begin{cases}H_{1-2 s}(x) \in Z & \text { for } 0 \leqq s \leqq \frac{1}{2}, \\
(f(x), 2-2 s) \in C Y & \text { for } \frac{1}{2} \leqq s \leqq 1 .\end{cases}
\end{aligned}
$$

Here, to simplify notations we omit all mention of a nullhomotopy $H_{t}$. With these definitions we assert

THEOREM 5. The connecting diagram above is commutative up to homotopy.

Proof. Let $G_{\tau}(0 \leqq \tau \leqq 1)$ be a homotopy $E_{f} \rightarrow E_{g}$ given by

$$
G_{\tau}(x, \beta)=\left(\beta(\tau), \gamma_{\tau}\right) \quad \text { for } x \in X, \beta \in E Y, f(x)=\beta(1),
$$

where

$$
\gamma_{\tau}(s)= \begin{cases}H_{(1-2 s+\tau)(1+\tau)^{-1}}(x), & 0 \leqq s \leqq 1+\tau / 2 \\ g \beta(2-2 s+\tau) & 1+\tau / 2 \leqq s \leqq 1\end{cases}
$$

Since $G_{0}(x, \beta)=\left(y_{0}, \eta_{f, g}(x, \beta)\right), G_{1}(x, \beta)=\xi_{f, g}(x)$, it follows that $I g \circ \eta_{f, g} \simeq$ $\xi_{f, g} \circ P f$.

Similarly, if we consider a homotopy $G_{\tau}^{\prime}: C_{f} \rightarrow C_{g}(0 \leqq \tau \leqq 1)$ defined by

$$
\begin{aligned}
G_{\tau}^{\prime}(y) & =(y, \tau) \\
G_{\tau}^{\prime}(x, s) & = \begin{cases}H_{(1-2 s+\tau)(1+\tau)-1}(x), & 0 \leqq s \leqq(1+\tau) / 2, \\
(f(x), 2-2 s+\tau), & (1+\tau) / 2 \leqq s \leqq 1,\end{cases}
\end{aligned}
$$

then we see that $\eta_{f, g}^{\prime} \circ Q f \simeq P^{\prime} g \circ \xi_{f, g}^{\prime}$. The other verifications are straightforward.

$\xi_{f, g}, \xi_{f, g}^{\prime}$, etc. will be called connecting maps in the sequel.

7.2. Next we shall determine to what extent the connecting maps are altered by the choice of nullhomotopies of $g \circ f$ or by alterations of $f, g$ within their homotopy classes. 
Let $\bar{\xi}_{f, g}, \bar{\xi}_{f, g}^{\prime}$, etc. be the connecting maps which are constructed by using another nullhomotopy $\bar{H}_{t}$ of $g \circ f$. It is clear that the correspondence

$$
(x, s) \rightarrow \begin{cases}\bar{H}_{1-2 s}(x), & 0 \leqq s \leqq \frac{1}{2} \\ H_{2 s-1}(x), & \frac{1}{2} \leqq s \leqq 1\end{cases}
$$

determines two maps $\kappa(\bar{H}, H): \mathrm{X} \rightarrow \Omega Z, \kappa^{\prime}(\bar{H}, H): S X \rightarrow Z$. It is readily verified that

$$
\begin{array}{ll}
\mu \circ\left\{\kappa(\bar{H}, H) \times \xi_{f, g}\right\} \simeq \bar{\xi}_{f, g}, & \mu \circ\left\{\kappa(\bar{H}, H) \times \eta_{f, g}\right\} \simeq \bar{\eta}_{f, g}, \\
\left\{\kappa^{\prime}(\bar{H}, H) \vee \xi_{f, g}^{\prime}\right\} \circ \mu^{\prime} \simeq \bar{\xi}_{f, g}^{\prime}, & \left\{\kappa^{\prime}(\bar{H}, H) \vee \eta_{f, g}^{\prime}\right\} \circ \mu^{\prime} \simeq \bar{\eta}_{f, g}^{\prime},
\end{array}
$$

where $\mu$ and $\mu^{\prime}$ are left operations in $E_{g}, C_{g}$ as defined in the previous section.

Secondly, when $\bar{f} \simeq f$, we shall construct $\xi_{\bar{f}}, g$, etc. by using the nullhomotopy $g \circ f_{t}$ followed by $H_{s}$, where $f_{t}$ is a homotopy connecting $\bar{f}$ with $f$. Then we see at once that $\xi_{\bar{f}, g} \simeq \xi_{f, g}, \eta_{\bar{f}, g}^{\prime} \simeq \eta_{f, g}^{\prime}$ and that the diagrams
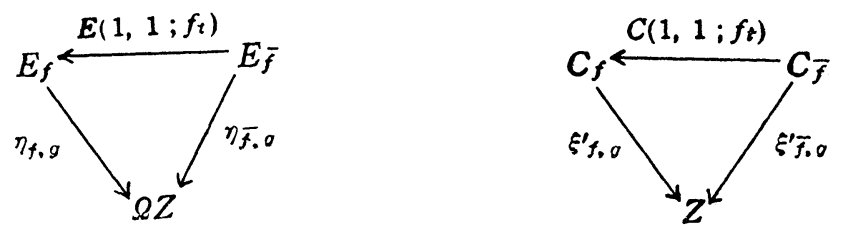

are homotopy commutative, where each horizontal map is a homotopy equivalence (cf. Lemmas 6,9). Similarly for $\bar{g} \simeq g$. Thus we have established

THEOREM 6. The effect of changing a nullhomotopy of $g \circ f$ upon connecting maps is described in terms of the left operation of some element in $\pi(X$, $\Omega Z)$ or $\pi(S X, Z)$. When $f$ and $g$ are altered with their homotopy classes, the resulting connecting maps are equivalent to the initial ones.

7.3. In case $g \circ f$ is just a constant, we may simplify the definition of connecting maps to some degree, i.e., in that case we set

$$
\begin{aligned}
& \xi_{f, g}(x)=\left(f(x), e_{z_{0}}\right), \quad \eta_{f, g}(x, \beta)=(g \beta)^{-1}, \\
& \xi_{f, g}^{\prime}(y)=g(y), \quad \xi_{f, g}^{\prime}(x, s)=z_{0}, \quad \eta_{f, g}^{\prime}(x, s)=(f(x), 1-s) .
\end{aligned}
$$

We shall now prove a result corresponding to excision theorems due to Eckmann and Hilton [4]

Theorem 7. i) If $g$ is a fibering with fibre $X$, then $\xi_{f, g}$ and $\eta_{f, g}$ are homo- 
topy equivalences. ii) Let $f$ be a cofibering with cofibre $Z$. Then we have homotopy equivalences $\xi_{f, g}^{\prime}$ and $\eta_{f, g}^{\prime}$.

Proof. The first halves of each assertion are just restatements of Th. 1 and Lemma 11 . Therefore it remains to prove that $\eta_{f, g}$ and $\eta_{f, g}^{\prime}$ are equivalences.

That $\eta_{f, g}$ is homotopic to the composite map

$$
E_{f} \stackrel{E\left(1, \xi_{f, g}\right)}{\longrightarrow} E_{P g} \stackrel{N g}{\longrightarrow} \Omega Z,
$$

where $\mathrm{Ng}$ is a map as defined in 2.2 , follows from the next computation

$$
\begin{aligned}
N g \circ E\left(1, \xi_{f, g}\right)(x, \beta) & =N g\left(\xi_{f, g}(x), \beta\right) \\
& =N g\left(\left(x, e_{z_{0}}\right), \beta\right) \\
& =N g\left(e_{z_{0}}, \beta\right)=e_{z_{0}} \cdot(g \beta)^{-1} .
\end{aligned}
$$

Since, by 2.2 and Lemma $6, E\left(1, \xi_{f, g}\right)$ and $N g$ are homotopy equivalences, so is $\eta_{f, g}$, which proves (i).

As regards $\eta_{f, g}^{\prime}$, consider the composite map

$$
S X \stackrel{\lambda^{\prime}}{\longrightarrow} C_{P^{\prime} f} \stackrel{C\left(1, \xi^{\prime}, g\right)}{\longrightarrow} C_{g}
$$

where $\lambda^{\prime}$ is the map determined by $\lambda^{\prime}: C_{f} \rightarrow M_{P^{\prime} f}$ in Lemma 12 . It is easy to show

$$
C\left(1, \xi_{f, g}^{\prime}\right) \circ \lambda^{\prime}(x, s)= \begin{cases}z_{0} \in Z, & 0 \leqq s \leqq \frac{1}{2} \\ (x, 2-2 s) \in C Y, & \frac{1}{2} \leqq s \leqq 1\end{cases}
$$

so that it is homotopic to $\eta_{f, g}^{\prime}$. One sees from Lemmas 9 and 11 that $\eta_{f, g}^{\prime}$ is a equivalence. This concludes our proof.

\section{Suspensions}

8.1. We shall give here a definition of suspensions for an arbitrary map $f: X \rightarrow Y$ which is substantially a generalization of usual ones, as mentioned in Remarks of 8.1 and 8.2

Before doing so we make a convention. $\pi(S X, Y)$ and $\pi(X, \Omega Y)$ are in $1-1$ correspondence with each other by the rule $\{\tilde{f}(x)\}(s)=f(x, s)$ for $\tilde{f}$ : $X \rightarrow \Omega Y, f: S X \rightarrow Y$. In this case we use the notation $[\tilde{f}] \leftrightarrow[f]$. 
Let $v: C_{f} \rightarrow Z$ be given, then we set $u=v \circ P^{\prime} f: \quad$ Note that $X \stackrel{f}{\rightarrow} Y \stackrel{u}{\longrightarrow} Z$ and $E_{f} \stackrel{P f}{\longrightarrow} X \stackrel{f}{\longrightarrow} Y$ are nullhomotopic triples. Thus we may consider the diagram

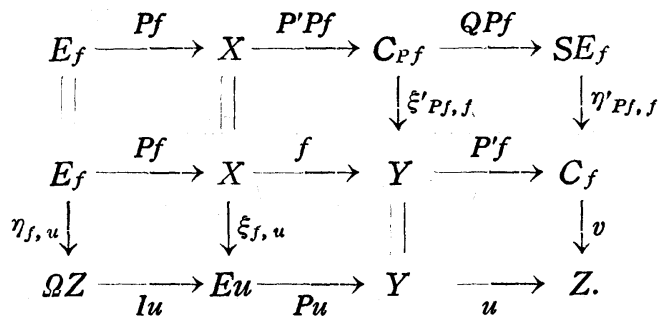

We define the Eilenberg-MacLane suspension for $f$

$$
\sigma^{*}: \pi\left(C_{f}, Z\right) \rightarrow \pi\left(S E_{f}, Z\right)
$$

by taking $\sigma^{*}([v])=\left[v \circ \eta_{P f, f}^{\prime}\right]$. We also define

$$
\bar{\sigma}^{*}: \pi\left(C_{f}, Z\right) \rightarrow \pi\left(E_{f}, \Omega Z\right)
$$

by $\bar{\sigma}^{*}([v])=\left[\eta_{f, u}\right] . \quad f=P u \circ \xi_{f, u}$ may be called the Postnikov factorization of $f$ with respect to $v$.

Since we have

$$
\begin{aligned}
& v \circ \eta_{P f, f}^{\prime}((x, \beta), s)=\left\{\begin{array}{lr}
u \beta(2 s), & 0 \leqq s \leqq \frac{1}{2}, \\
v(x, 2-2 s), & \frac{1}{2} \leqq s \leqq 1,
\end{array}\right. \\
& \left\{\eta_{f, u}(x, \beta)\right\}(s)= \begin{cases}v(x, 2 s), & 0 \leqq s \leqq \frac{1}{2}, \\
u \beta(2-2 s), & \frac{1}{2} \leqq s \leqq 1,\end{cases}
\end{aligned}
$$

we obtain

Lemma 14. $\bar{\sigma}^{*}([v]) \leftrightarrow-\sigma^{*}([v])$.

Remark. In case $f$ is a fibering with fibre $F$, we shall call

$$
\left(\eta_{P f, f}^{\prime} \circ S \Phi\right)^{*}: \pi\left(C_{f}, Z\right) \rightarrow \pi(S F, Z)
$$

the suspension of the fibering $f$, where $\Phi: F \rightarrow \mathrm{E}_{f}$ is an equivalence given in Th. 1. In particular, let $f$ be the Serre fibering $E Y \rightarrow Y$ defined by $\beta \rightarrow \beta(1)$ and let $u: Y \rightarrow Z$ be given; then we set $v(y)=u(y), v(\beta, s)=u \beta(s)$ for $y \in Y$, 
$\beta \in E Y, 0 \leqq s \leqq 1$, obtaining a map $v: C_{f} \rightarrow Z$. We see then that $\eta_{f, u} \circ \emptyset \simeq \Omega u$ : $\Omega Y \rightarrow \Omega Z$, where $\Phi: \Omega Y \rightarrow E_{f}$ is an equivalence.

8.2. Given a map $v^{\prime}: Z \rightarrow E_{f}$, we set $u^{\prime}=P f \circ v^{\prime}$ and we proceed in a dual fashion. Consider the diagram

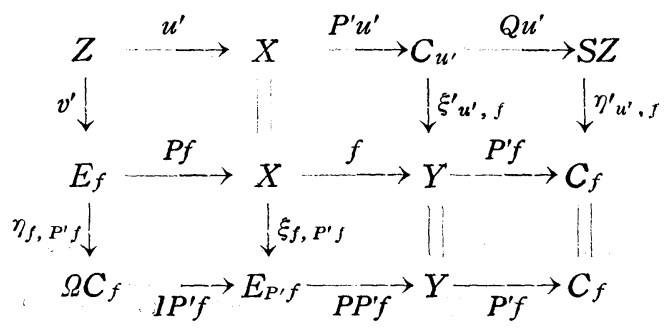

$f=\xi_{u^{\prime}, f}^{\prime} \circ P^{\prime} u^{\prime}$ may be said to be the Moore factorization of $f$ with respect to $v^{\prime}$.

We define the Freudenthal suspension for $f$

$$
\sigma_{*}: \pi\left(Z, E_{f}\right) \rightarrow \pi\left(Z, \Omega C_{f}\right)
$$

by setting $\sigma_{*}\left(\left[v^{\prime}\right]\right)=\left[\gamma_{f, p^{\prime} f} \circ v^{\prime}\right]$. We define also

$$
\bar{\sigma}_{*}: \pi\left(Z, E_{f}\right) \rightarrow \pi\left(S Z, C_{f}\right)
$$

by $\bar{\sigma}_{*}\left(\left[v^{\prime}\right]\right)=\left[\eta_{u^{\prime}, f}^{\prime}\right]$. As in the case of the Eilenberg-MacLane suspension, we may obtain

Lemma $14^{\prime} . \quad \bar{\sigma}_{*}\left(\left[v^{\prime}\right]\right) \leftrightarrow-\sigma_{*}\left(\left[v^{\prime}\right]\right)$.

Remark. In case in which $f$ is a cofibering with cofibre $Y / X$, then we have a natural equivalence $\Phi^{\prime}: C_{f} \rightarrow Y / X$. (Lemma 11). We say that

$$
\left(\Omega \Phi^{\prime} \circ \eta f, p^{\prime} f\right)_{*}: \pi\left(Z, E_{f}\right) \rightarrow \pi(Z, \Omega(Y / X))
$$

is the suspension of the cofibering $f$. In particular consider $f: X \rightarrow C X$ which is the injection. Given $u^{\prime}: Z \rightarrow X$, we define $v^{\prime}: Z \rightarrow E_{f}$ by $v^{\prime}(z)=\left(u^{\prime}(z), \beta^{\prime}\right)$ where $\beta^{\prime}: I \rightarrow C X$ is defined by $\beta^{\prime}(s)=\left(u^{\prime}(z), s\right), 0 \leqq s \leqq 1$. It follows at once that $\Phi^{\prime} \circ \eta_{u^{\prime}, f}^{\prime} \simeq S u^{\prime}$, where $\Phi^{\prime}: C_{f} \rightarrow S X$ is a natural equivalence.

8.3. We shall now establish naturality of suspensions. Let

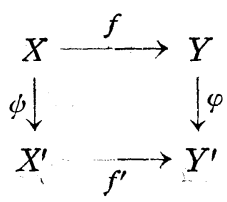


be a transformation as in 6.1 . It will be reasonable to consider the diagrams
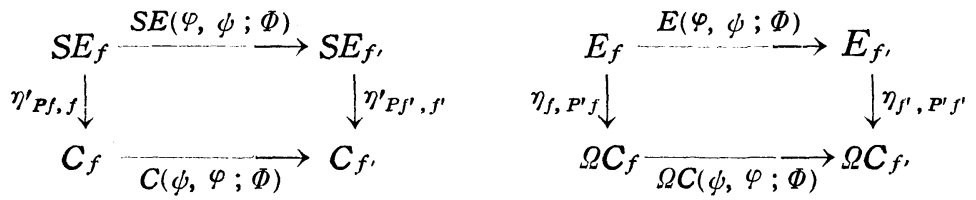

The following lemma is readily deduced from the definitions involved.

Lemma 15. The above diagrams (7) are homotopy commutative.

Next, given maps $\bar{v}: C_{f} \rightarrow Z, \widetilde{v}: Z \rightarrow E_{f}$, we set

$$
\begin{aligned}
& v=\bar{v} \circ C(\phi, \varphi ; \emptyset), \quad \bar{u}=\bar{v} \circ P^{\prime} f^{\prime}, \quad u=v \circ P^{\prime} f, \\
& v^{\prime}=E(\varphi, \psi ; \Phi) \circ \widetilde{v}, \quad \tilde{u}=P f \circ \widetilde{v}, \quad u^{\prime}=P f^{\prime} \circ v^{\prime} .
\end{aligned}
$$

Consider the diagrams

(8)
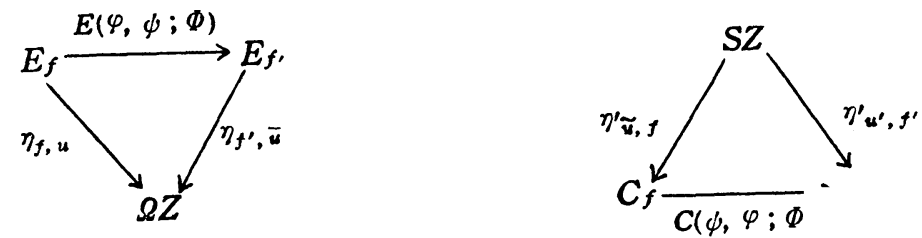

Observing that $u=\bar{u} \circ \varphi, u^{\prime}=\psi \circ \widetilde{u}$, we easily verify

Lemma 16. The diagrams (8) are homotopy commutative.

\section{Suspension theorems}

9.1. We are now in a position to prove an important property concerning suspensions, which is an extension of usual suspension theorems. In the rest of this paper we assume that the spaces to be considered are 1-connected.

Theorem 8. Let $f: X \rightarrow Y$ be any map and suppose $Y$ is $r$-connected, $E_{f}$ s-connected. Then

$$
\sigma^{*}: H^{q}\left(C_{f}\right) \rightarrow H^{q}\left(S E_{f}\right)=H^{q-1}\left(E_{f}\right)
$$

is an isomorphism for $q \leqq r+s+1$ and a monomorphism for $q=r+s+2$.

Proof. In view of Lemmas 13, 15, 6, 9, we may restrict our attention to the case in which $f: X \rightarrow Y$ is an inclusion cofibering. In this case

$$
E_{f}=E_{y_{0}, x}(Y), \quad C_{f}=C X \cup Y,
$$


and $\eta_{P f, f}^{\prime}$ is given by

$$
\eta_{P f, f}^{\prime}(\beta, s)= \begin{cases}\beta(2 s) \in Y, & 0 \leqq s \leqq \frac{1}{2}, \beta \in E_{f}, \\ (\beta(1), 2-2 s) \in C X, & \frac{1}{2} \leqq s \leqq 1, \beta \in E_{f} .\end{cases}
$$

We shall form a diagram

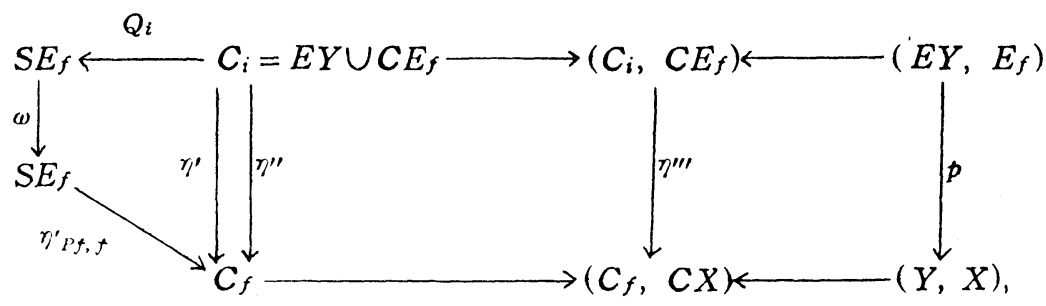

where $\omega$ is the involution determined by inversion of suspension parameter; $i: E_{f} \rightarrow E Y$ the inclusion; $\eta^{\prime}$ the composite $\eta_{P f, f}^{\prime} \circ \omega \circ Q i ; \eta^{\prime \prime}$ the map given by $\eta^{\prime \prime}(\beta)=\beta(1)$ for $\beta \in E Y, \eta^{\prime \prime}(\beta, s)=(\beta(1), s)$ for $(\beta, s) \in C E_{f} ; \eta^{\prime \prime \prime}$ is induced by $\eta^{\prime} ; p$ the map defined by $p(\beta)=\beta(1)$ for $\beta \in E Y$; other horizontal maps are all inclusions.

Define $G_{t}(0 \leqq t \leqq 1)$ to be the homotopy such that

$$
\begin{aligned}
& G_{t}(\beta)=\beta(t) \quad \text { for } \beta \in E Y, \\
& G_{t}(\beta, s)=\left\{\begin{array}{lll}
\left(\beta(1), \frac{2 s}{1+t}\right), & 0 \leqq s \leqq \frac{1+t}{2}, & \beta \in E_{f}, \\
\beta(2-2 s+t), & \frac{1+t}{2} \leqq s \leqq 1, & \beta \in E_{f} .
\end{array}\right.
\end{aligned}
$$

Since $G_{0}=\eta^{\prime}, G_{1}=\eta^{\prime \prime}$, we have $\eta^{\prime} \simeq \eta^{\prime \prime}$. We see at once that the diagram above is commutative.

By passing to cohomolgy it is clear that all horizontal maps, $Q i$ and $\omega$ induce isomorphisms. Note that $p$ is a fibre map. Since $\Omega Y$ is $(r-1)$-connected and $(Y, X)$ is $(s+1)$-connected, it follows from a well known theorem ([14], Th. 1.B) that $p^{\prime}: H^{q}(Y, X) \rightarrow H^{q}\left(E Y, E_{f}\right)$ is isomorphic for $q \leqq r+s+1$ and monomorphic for $q=r+s+2$, so that the same is true for $\sigma^{*}=\left(\eta_{P f, f}^{\prime}\right)^{*}$, which is what we wanted to prove.

Theorem 8'. Let $f: X \rightarrow Y$ be any map, and let $X$ and $E_{f}$ be $r$ - and s-connected respectively. Then 


$$
\sigma_{*}: \pi_{q}\left(E_{f}\right) \rightarrow \pi_{q}\left(\Omega C_{f}\right)=\pi_{q+1}\left(C_{f}\right)
$$

is an isomorphism for $q \leqq r+s$ and an epimorphism for $q=r+s+1$.

Proof. As before we may assume that $f$ is an inclusion. We consider the diagram

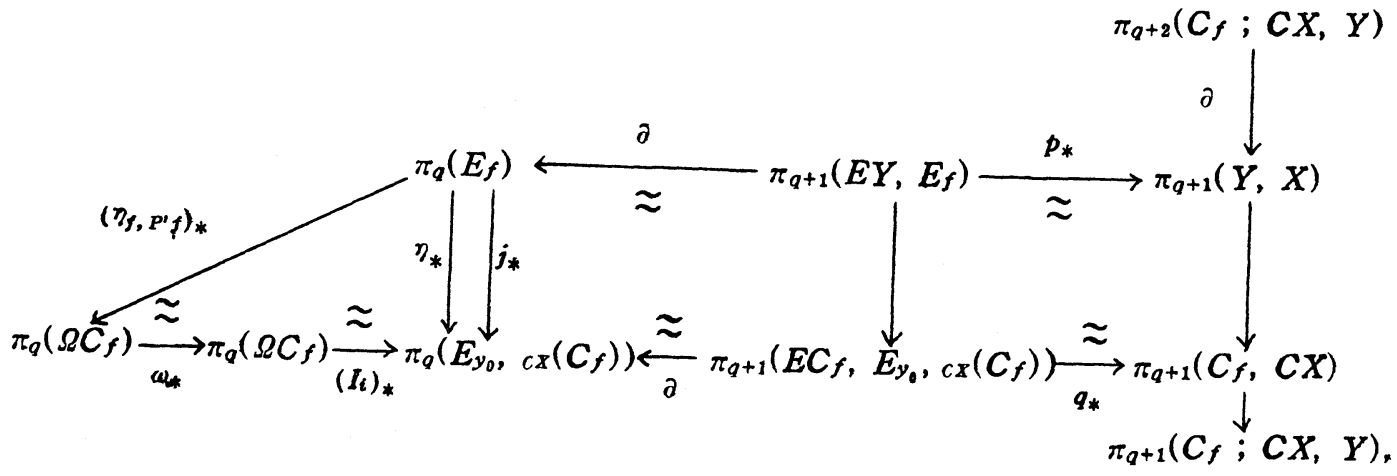

where $\omega$ is the involution induced by inverting loops; $\eta=(l i) \circ \omega \circ \gamma_{f, p, f}, i$ : $C X \rightarrow C_{f}$ the inclusion; $p$ and $q$ are fibre maps defined by taking the terminal point of paths; other vertical maps are induced by injections.

By proceeding as in the previous theorem, we see that $\eta$ and $j$ are homotopic to each other. Since $(C X, X)$ is $(r+1)$-connected and $(Y, X)(\mathrm{s}+1)$ connected, we have

$$
\pi_{q+2}\left(C_{f} ; C X, Y\right)=0 \quad \text { for } q \leqq r+s
$$

by the triad theorem [2]. Hence it follows that $\sigma_{*}=\left(\eta f, p^{\prime} f\right)_{*}$ is isomorphic for $g \leqq r+s$ and onto for $q=r+s+1$. This completes the proof.

\section{Postnikov decomposition}

Let $f: X \rightarrow Y$ be any map such that $\pi_{q}\left(E_{f}\right)=0$ for $q \leqq n-1$. We abbreviate $\pi_{q}\left(E_{f}\right)$ by $\pi_{q}$. By a convention made in 9.1 we have $\pi_{1}(Y)=0$, so that Th. 8 asserts that $\bar{\sigma}^{*}: H^{q}\left(C_{f}\right) \rightarrow H^{q-1}\left(E_{f}\right)$ is isomorphic for $q \leqq n+1$. Therefore we can find a map

$$
v: C_{f} \rightarrow K\left(\pi_{n}, n+1\right)
$$

such that $\bar{\sigma}^{*}([v])=\left[\eta_{f, u}\right] \in \pi\left(E_{f}, \Omega K\left(\pi_{n}, n+1\right)\right)=H^{n}\left(E_{f}\right) \approx \operatorname{Hom}\left(H_{n}\left(E_{f}\right), \pi_{n}\right)$ is the inverse of Hurewicz isomorphism, in which we set $u=v \circ P^{\prime} f$. 
We have the maps

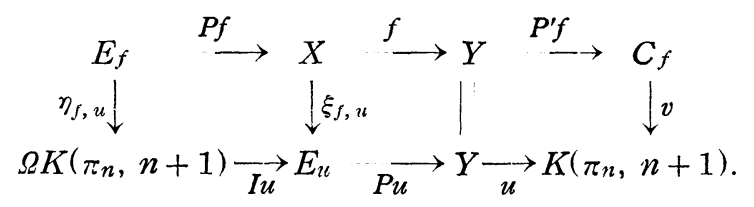

Since the relative mapping sequence for the triple $f=P u \circ \xi_{f, u}$ is written

$$
\begin{gathered}
\Omega E_{f} \stackrel{\Omega k}{\longrightarrow} \Omega E_{P u} \longrightarrow E_{\xi_{f}, u} \longrightarrow E_{f} \stackrel{k}{\longrightarrow} E_{P u} \\
K\left(\pi_{n}, n-1\right)
\end{gathered}
$$

it follows from Th. 3 that $\pi_{q}\left(E_{\xi_{f}, u}\right) \approx \pi_{q}\left(E_{f}\right)$ for $q \neq n, n-1$. A simple computation shows that $\eta_{f, u}$ coincides with the composite

$$
E_{f} \stackrel{k}{\longrightarrow} E_{P u} \stackrel{N u}{\longrightarrow} \Omega K\left(\pi_{n}, n+1\right)
$$

where $N_{u}$ is an equivalence constructed in 2.2 , and thus we have

$$
k_{*}: \pi_{n}\left(E_{f}\right) \approx \pi_{n}\left(E_{P u}\right),(\Omega k)_{*}: \pi_{n-1}\left(\Omega E_{f}\right) \approx \pi_{n-1}\left(\Omega E_{P u}\right) .
$$

These results show that

$$
\begin{cases}\pi_{q}\left(E_{\xi_{f}, u}\right)=0 & \text { for } q \leqq n \\ \pi_{q}\left(E_{\xi_{f}, u}\right) \approx \pi_{q}\left(E_{f}\right) & \text { for } q \geqq n+1\end{cases}
$$

On the other hand, we obtain quickly from $\left(\mathfrak{M} \xi_{f, u}\right)_{*}$ that

$$
\begin{cases}\left(\xi_{f, u}\right)_{*}: \pi_{q}(X) \approx \pi_{q}\left(E_{u}\right) & \text { for } q \leqq n, \\ \left(\xi_{f, u}\right)_{*}: \pi_{n+1}(X) \rightarrow \pi_{n+1}\left(E_{u}\right) & \text { onto. }\end{cases}
$$

Furthermore we have

$$
(P u)_{*}: \pi_{q}\left(E_{u}\right) \approx \pi_{q}(Y) \quad \text { for } q \neq n+1, n .
$$

This construction is essentially due to Eckmann and Hilton [6]. They call it the homotopy decomposition of $f$.

In case $f$ is a fibering then, by Th. $1, E_{f}$ is equivalent to the fibre of $f$, and thus we see from (9) that we have the Moore-Postnikov system for $f$ ([1], p. 911). In particular, when $Y$ is a point we obtain the Postnikov system for the space $X$.

It $X$ is a point then $E_{f}=\Omega Y$, so that $P u: E_{u} \rightarrow Y$ is a fibering in which 
the fibre is $K\left(\pi_{n+1}(Y), n\right)$ and moreover $P u$ is $(n+1)$-connective by (10), (11). This is nothing but the Cartan-Serre-Whitehead technique for killing homotopy groups [3].

\section{Functional operations}

11.1. In this section we try to make an explicit deduction of the formulas stated in [10], without making use of the universal example.

Suppose given a quadruple $L \stackrel{f}{\rightarrow} K \stackrel{h}{\rightarrow} X \stackrel{\theta}{\rightarrow} Y$ such that $h \circ f \simeq 0, \theta \circ h \simeq 0$ which are realized by homotopies $H_{t}, G_{t}$ respectively. Using the diagram

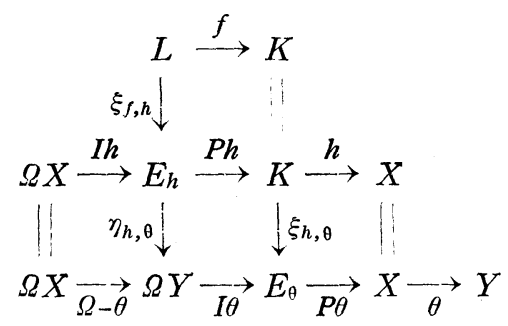

we shall define the functional $\theta$-operation $\bar{\theta}_{f}$ by

$$
\vec{\theta}_{f}(h)=\left[\eta_{h, \theta} \circ \xi_{f, h}\right] .
$$

This must be considered as an element of the set of equivalence classes by left operation of $f^{*} \pi(K, \Omega Y)$ and right operation of $(\Omega \theta)_{*} \pi(L, \Omega X)$.

Alternatively we consider the maps

$$
\begin{aligned}
& L \stackrel{f}{\rightarrow} K \stackrel{P^{\prime} f}{\longrightarrow} C_{f} \stackrel{Q f}{\longrightarrow} S L \stackrel{S-f}{\rightarrow} S K \\
& \downarrow \xi_{f, h}^{\prime} \quad \downarrow^{\eta_{f, h}^{\prime}} \\
& K \stackrel{h}{\rightarrow} X \stackrel{P^{\prime} h}{\rightarrow} C_{h} \stackrel{Q h}{\longrightarrow} S K \\
& \downarrow \xi^{\prime} h, \theta \\
& X \underset{\theta}{\longrightarrow} Y
\end{aligned}
$$

and we set

$$
\theta_{f}(h)=\left[\xi_{h, \theta}^{\prime} \circ \eta_{f, h}^{\prime}\right]
$$

which is regared as an element of $\pi(S L, Y)$ classified by right operation of $(S f)^{*} \pi(S K, Y)$ and left operation of $\theta^{*} \pi(S L, X)$.

The following gives a relationship between the above two definitions.

Proposition 7. $\bar{\theta}_{f}(h) \leftrightarrow-\theta_{f}(h) \quad$ (cf. Th. 5.1 in [10]) 
Proof. By a direct calculation based on the definition of connecting maps we have

$$
\begin{aligned}
& \left\{\eta_{h, \theta} \circ \xi_{f, h}(x)\right\}(s)=\left\{\begin{array}{lll}
G_{1-2 s}(f(x)), & 0 \leqq s \leqq \frac{1}{2}, & x \in L, \\
\theta H_{2 s-1}(x), & \frac{1}{2} \leqq s \leqq 1, & x \in L,
\end{array}\right.
\end{aligned}
$$

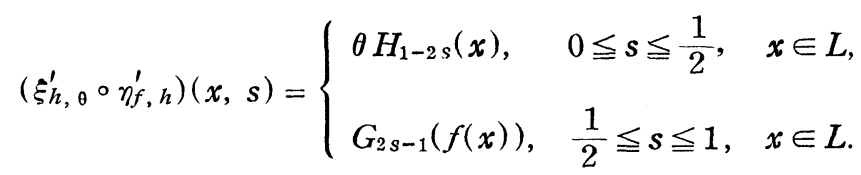

Our assettion follows from these.

11.2. Let $f, h, \theta$ be as above and let $\phi: E \rightarrow Z$ be given; then we set $\theta^{\prime}=\phi \circ(1 \theta): \Omega Y \rightarrow Z$. Note that $\theta^{\prime} \circ \Omega \theta \simeq 0$ by the exactness of $(\mathfrak{M} \theta)_{*}$.

Following Peterson [10] we shall define the secondary $\theta$-operation $\Phi_{\theta}$ determined by $\phi$ as follows :

$$
\Phi_{\theta}(h)=\left[\phi \circ \xi_{h, 0}\right],
$$

which is regarded as an element of $\pi(K, Z)$ classified by $\phi$-image of left operation of $\pi(K, \Omega Y)$. Thus, in order to describe $\Phi_{\theta}$ completely, we need more explicit information about $\phi^{\circ} \mu$, where $\mu: \Omega Y \times E_{\theta} \rightarrow E_{\theta}$ is left operation.

From the above definitions we see that

$$
\theta^{\prime} \circ \eta_{h, \theta} \circ \xi_{f, h}=\phi \circ(I \theta) \circ \eta_{h, \theta} \circ \xi_{f, h}=\phi \circ \xi_{h, \theta} \circ f .
$$

Therefore we have proved

Proposition 8. (cf. Th. 7.1 in [10]) $\theta^{\prime}\left(\bar{\theta}_{f}(h)\right)=f^{*} \Phi_{\theta}(h) \bmod \theta^{\prime} f^{*} \pi(K, \Omega Y)$ $+f^{*} \phi$ (left operation of $\pi(K, \Omega Y)$ ).

11.3. Next, let $f: L \rightarrow K$ be a fibering with fibre $F$, and let $i: F \rightarrow L$ be the inclusion. Suppose $h: K \rightarrow X$ is such that $h \circ f \simeq 0$ by a homotopy $H_{t}$ $(0 \leqq t \leqq 1)$, and let $\psi: X \rightarrow Y$ be a map with $\phi \circ h \simeq 0$. Then we have the diagram 


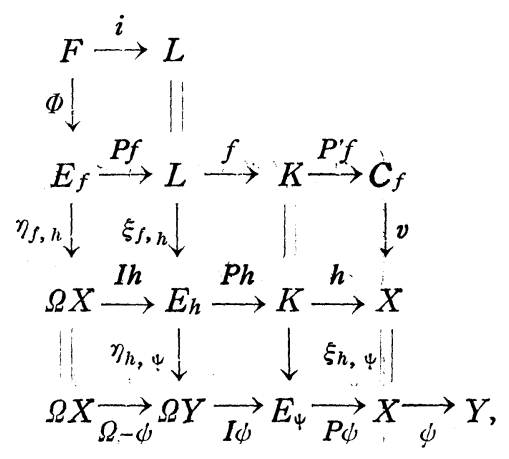

where $\Phi$ is a natural equivalence (cf. Th. 1) and $v$ is defined by

$$
\begin{aligned}
v(k)=h(k) & \text { for } k \in K, \\
v(l, s)=H_{1-s}(l) & \text { for } l \in L, 0 \leqq s \leqq 1 .
\end{aligned}
$$

Since $\bar{\sigma}^{*}([v])=\left[\eta_{f, h} \circ \Phi\right]$ in the fibering $f$ (cf. 8.1, Remark), it follows from the homotopy commutativity of the diagram

Proposition 9. (cf. [10], Lemma 6.2) $i^{*} \bar{\psi}_{f}(h)=-(\Omega \psi)_{*} \bar{\sigma}^{*}([v]) \bmod$ $(\Omega \psi)_{*} \pi(L, \Omega X)$.

11.4. Let $L \stackrel{f}{\rightarrow} K \stackrel{h}{\rightarrow} X \stackrel{\theta}{\rightarrow} Y \stackrel{\phi}{\rightarrow} Z$ be maps such that there exist homotopies $H_{t}: \theta \circ h \circ f \simeq 0, G_{t}: \psi \circ \theta \simeq 0(0 \leqq t \leqq 1)$. Then we can easily verify that both $\eta_{\theta, \psi} \circ \xi_{h \circ f, \theta}$ and $\eta_{\theta \circ h, \psi} \circ \xi_{f, \theta \circ h}$ are given by

$$
(x, s) \rightarrow\left\{\begin{array}{lll}
G_{1-2 s}(h f(x)), & 0 \leqq s \leqq \frac{1}{2}, & x \in L, \\
\psi H_{2 s-1}(x), & \frac{1}{2} \leqq s \leqq 1, & x \in L .
\end{array}\right.
$$

Hence we have shown

Proposition 10. $\bar{\psi}_{h \circ f}(\theta)=\bar{\psi}_{f}(\theta \circ h)$.

In case $\bar{\sigma}^{*}: \pi\left(C_{0}, Z\right) \rightarrow \pi\left(E_{\theta}, \Omega Z\right)$ is onto, then there exists a map $\phi: Y \rightarrow Z$ such that $\left[\eta_{\theta, \psi}\right]=[\phi]$ for any $\phi: E_{\theta} \rightarrow \Omega Z$, and then we have $(I \theta)^{*}[\phi]=-[\Omega \psi]$. We deduce from Prop. 10.

Corollary. $\quad \Phi_{\theta}(h \circ f)=\bar{\psi}_{f}(\theta \circ h) \bmod (\Omega \psi)_{*} \pi(L, \Omega Y)+f^{*} \pi(K, \Omega Z) \quad(\mathrm{cf}$. [10], Th. 6.3). 


\section{REFERENCES}

[1] W. D. Barcus and P. J. Meyer, The suspension of a loop space, Amer. J. Math., 80 (1958), 895-920.

[2] A. L. Blakers and W. S. Massey, The homotopy groups of a triad II, Ann. of Math., 55 (1952), 192-201.

[ 3 ] H. Cartan and J. P. Serre, Espaces fibrés et groupes d'homotopie I. Constructions générales, C. R. Acad. Sci. Paris, 234 (1952), 288-290.

[4] B. Eckmann and P. J. Hilton, Groupes d'homotopie et dualité. Groupes absolus, C. R. Acad. Sci. Paris, 246 (1958), 2444-2447; Suites exactes, ibid. 2555-2558.

[5] B. Eckmann and P. J. Hilton, Transgression homotopique et cohomologique, ibid., 247 (1958), 620-623.

[6] B. Eckmann and P. J. Hilton, On the homology and homotopy decomposition of continuous maps, Proc. Nat. Acad. Sci. U.S.A., 45 (1959), 372-375.

[7] W. Hurewicz, On the concept of fibre space, Proc. Nat. Acad. Sci. U.S.A., 41 (1955), 956-961.

[8] I. M. James and J. H. C. Whitehead, Note on fibre spaces, Proc. London Math. Soc., 4 (1954), 129-137.

[9] W. S. Massey, Homotopy groups of triads, Proc. International Congress of Math. Cambridge, Mass. U.S.A., (1950), 371-382.

[10] F. P. Peterson and N. Stein, Secondary cohomology operations: two formulas, Amer. J. Math., 81 (1959), 281-305.

[11] F. P. Peterson and E. Thomas, A note on non-stable cohomology operations, Boletin de la Sociedad Mathematica in Mexicana, segunda serie, 3 (1958), 13-18.

[12] D. Puppe, Homotopiemengen und ihre induzierte Abbildungen I, Math. Z., 69 (1958), 299-344; II, ibid., 395-417.

[13] H. Samelson, Groups and spaces of loops, Comment. Math. Helv., 28 (1954), 278-287.

[14] J. -P. Serre, Groupes d'homotopie et classes de groupes abéliens, Ann. of Math., 58 (1953), 258-294.

[15] E. H. Spanier and J. H. C. Whitehead, On fibre spaces in which the fibre is contractible, Comment. Math. Helv., 29 (1955), 1-8.

[16] M. Sugawara, On a condition that a space is an H-space, Math. J. Okayama Univ., 6(1957), 109-129.

[17] J. H. C. Whitehead, Combinatorial homotopy I, Bull. Amer. Math. Soc., 55 (1949), 245-277.

Department of Mathematics

Shizuoka University

Shizuoka, Japan 